\title{
6. Der Untersuchungsgegenstand: Bewegungsmedien
}

Bewegungsinterne Medien sind ein konstitutiver Bestandteil von Protesten, sozialen Bewegungen und Subkulturen. Sie werden auch als »'Lebenselixier $<$ im >Kampf um Öffentlichkeit « bezeichnet $^{1}$ und haben die Funktion, alternative oder Gegenöffentlichkeiten herzustellen: Als »Alternativmedien« produzieren sie eigenes Wissen und als »Gegenmedien « positionieren sie sich gegen eine hegemoniale Öffentlichkeit. Eine weitere - und Oliver Marchart zufolge die relevantere - Funktion von bewegungsinternen Medien ist die dauerhafte Stabilisierung einer »bewegungseigenen politischen oder gegenkulturelle[n] Identität «. ${ }^{2}$ Judith Butler zufolge sind Medien selbst ein wesentlicher Bestandteil des kollektiven Wir, das durch sie hergestellt und stabilisiert wird:

»Insofern das Volk durch ein komplexes Zusammenspiel von Performanz, Bild, Akustik und all den anderen Techniken, die bei solchen Produktionen eine Rolle spielen, konstituiert wird, berichten die >Medien hauptet, sondern sind selbst ein ganz entscheidender Teil von dessen Definition. Sie unterstützen und ermöglichen diese Definition nicht nur, sondern sie sind der Stoff der Selbstkonstitution, der Ort des hegemonialen Kampfes darum, wer >wir « sind. $\aleph^{3}$

Auch die fünf für die vorliegende Untersuchung ausgewählten queeren Projekte nutzen allesamt bewegungsinterne Medien, um alternative und Gegenöffentlichkeiten, aber auch ein kollektives Wir herzustellen. Vor allem aufgrund der zentralen Rolle, die Medien für die Herstellung einer bewegungseigenen Identität spielen, wurden die bewegungsinternen Medien der fünf queeren Projekte als Material für die diskursanalytische Untersuchung ausgewählt. Bezug nehmend auf das von Jäger vorgeschlagene methodische Vorgehen, soll im Folgenden die Erstellung des Materialkorpus - auch »Archiv« genannt - beschrieben werden. ${ }^{4}$

\footnotetext{
O. Marchart: Die Prekarisierungsgesellschaft, S. 196.

Ebd., S. 197.

J. Butler: Anmerkungen zu einer performativen Theorie der Versammlung, S. 31.

S. Jäger: Kritische Diskursanalyse, S. 91.
} 


\section{Die Erstellung des Materialkorpus}

Gemeinsam ist allen fünf Projekten, dass die von ihnen produzierten bewegungsinternen Medien wichtige Orte des Austauschs und der Intervention sind. Es wurden mit wenigen Ausnahmen die gesamten zwischen 2010 und $2016^{5}$ produzierten projekteigenen Medien in den Materialkorpus aufgenommen. Nicht in den Materialkorpus aufgenommen wurden: erstens wissenschaftliche Studien, wie sie beispielsweise LesMigraS oder das Jugendnetzwerk Lambda BB veröffentlichen, weil aufgrund des speziellen Genres verhältnismäßig wenig Erkenntnisse für die Fragestellung erwartet wurden; zweitens Social-Media-Auftritte der fünf Projekte auf Plattformen wie Instagram, Facebook oder Twitter, nachdem in einem Beobachtungszeitraum von einem halben Jahr keine für die Fragestellung Erkenntnisse versprechende Veröffentlichungen stattgefunden haben; ${ }^{6}$ und drittens Podcasts, Radiosendungen und Videos, weil diese Medien zumeist nur von jeweils einem Projekt genutzt wurden.

Für die Erstellung des Materialkorpus wurden alle von den fünf Einrichtungen im Analysezeitraum produzierten politischen Stellungnahmen, Pressemitteilungen, Redebeiträge, Zeitschriften, Artikel, Broschüren, Flyer, Plakate und Website-Texte gesammelt. Hierzu wurden die fünf Projekte bereits im Zuge der ersten Kontaktaufnahme um Informationen über den Umfang und die Zugänglichkeit des Materials gebeten. Die Einrichtungen haben die meisten Zeitschriften, Flyer und Broschüren per Post zugeschickt. Pressemitteilungen, Redebeiträge, Artikel und Broschüren konnten größtenteils auf den projekteigenen Homepages heruntergeladen werden. Einige Texte mussten auch kopiert, fotografiert und abgespeichert werden. Im Fall der Türkis Rosa Lila Villa hat auch ein Archiv-Besuch in der Villa stattgefunden, der zusätzliche Materialien wie Broschüren, Flyer und Plakate erbrachte. Zudem wurden die meisten Interviews in den Räumen der Projekte geführt. So konnte auch in diesen Räumen Material gesichtet und gesammelt werden. Insgesamt wurden ca. 850 Seiten (Zeitschriften-)Artikel, 150 Seiten Stellungnahmen, Pressemitteilungen und Redebeiträge, 80 Seiten Website-Texte, ca. 200 Seiten Broschüren und Informationsmaterialien sowie ca. 50 Seiten Flyer und Plakate in den Materialkorpus aufgenommen. Der sehr umfangreiche Materialkorpus zeichnet sich dadurch aus, dass er sehr viele verschiedene größere und kleinere Trägermedien (Zeitschriften, Websites, Flyer, Broschüren und Plakate) sowie einzelne Textsorten (Artikel, Pressemitteilungen, Stellungnahmen und Redebeiträge), die wiederum in unterschiedlichen internen wie externen Organen veröffentlicht wurden, umfasst. Hinzu kommt, dass die Projekte jeweils unterschiedliche Medien nutzen und je nach Arbeitsschwerpunkt auch spezifische Themensetzungen und Zielgruppen haben. Um die später folgende Analyse zu kontextualisieren und zu informieren, sollen im Folgenden erstens die unterschiedlichen Trägermedien und Textsorten genrespezifisch eingeordnet werden und soll zweitens ein Einblick in das Material (Inhalte, Funktionen und Zielgruppen) der jeweils projektspezifischen Öffentlichkeiten gegeben werden. Ersteres

5 Die zeitliche Eingrenzung des Analysezeitraums wird in Kapitel fünf begründet.

6 Es wurden hauptsächlich Veranstaltungshinweise und Links von externen Blogs, Zeitungen oder Vereinen geteilt oder Pressemitteilungen und Flyer veröffentlicht, die ohnehin in den Materialkorpus aufgenommen wurden. 
ist unter anderem deswegen relevant, weil die von den Projekten produzierten Medien und Textsorten selten Gegenstand von (Diskurs-)Analysen sind und es daher wenig Wissen zu ihnen gibt. Eine genrespezifische Einordnung der unterschiedlichen Medien hat aber auch die Funktion, die Analyse zu informieren, um so zu verhindern, dass den Ergebnissen der Feinanalyse lediglich genrespezifische Unterschiede zugrunde liegen.

\subsection{Eine genrespezifische Einordnung der projekteigenen Medien}

Die in der vorliegenden Arbeit verwendeten Trägermedien und Textsorten sind sowohl in der sozialen Bewegungsforschung als auch in den Medien- und Sprachwissenschaften wenig erforscht und ausgearbeitet. Das hängt auch damit zusammen, dass dort "Protestöffentlichkeiten «, genauso wie hegemoniale Öffentlichkeiten, fast ausschließlich im Bereich der Massenmedien betrachtet werden. Viele protesteigene Medien wie beispielsweise Flyer oder Broschüren gelten in den Medien- und Kommunikationswissenschaften meistens nur als Zusatz zu den Massenmedien und finden daher kaum Eingang in die (Medien-)Forschung. ${ }^{7}$ Im $»$ Handbuch zur Entwicklung der Medien und Kommunikationsformen « werden beispielsweise Flugblätter und Flugschriften historisch kontextualisiert, Broschüren oder Flyer - die als moderne Form von Flugblättern und Flugschriften gelten - bleiben allerdings unerwähnt. Die Zeitschrift, als ein Medium, das immer noch weit verbreitet ist, ist dementgegen umfangreicher ausgearbeitet. Aber auch hier findet sich zumindest in den Medien- und Kommunikationswissenschaften wenig zur speziellen Rolle von Zeitschriften in sozialen Bewegungen. Feministische Forschungen haben vor allem für die zweite Frauenbewegung die Rolle von Zeitschriften zur Herstellung alternativer Öffentlichkeiten hervorgehoben. Hier werden neben den Inhalten und der spezifischen Rolle der jeweiligen Zeitschriften in der Bewegung vor allem institutionelle Rahmenbedingungen wie die hohe Fluktuationsrate und die Konflikte in der Redaktion oder die prekären Produktionsbedingungen thematisiert. ${ }^{8}$ Das Plakat wird in den Medienwissenschaften vergleichsweise ausführlich beschrieben und charakterisiert. Das liegt daran, dass dem Plakat lange Zeit eine wichtige Rolle in der Werbung und der Parteien-Politik zugekommen ist und es bis heute Werbe- sowie Wahlkampagnen in anderen (Massen-)Medien unterstützt. Literatur zu den Textsorten Pressemitteilung, Stellungnahme und Redebeiträge findet sich mitunter in der Politolinguistik, vor allem aber im Bereich der Public Relations. In der Politolinguistik beziehen sich die genrespezifischen Einordnungen allerdings auf ihre Funktion innerhalb klassischer Parteien-Politiken. Vereinzelt gibt es im Bereich der Public Relations Hinweise auf die spezielle Funktion von Pressemitteilungen und Stellungnahmen in Non-Profit-Organisationen wie NGOs, Vereinen oder Netzwerken.

7 O. Marchart: Die Prekarisierungsgesellschaft, S. $196 f$.

8 Vgl. Gruppe Feministische Öffentlichkeit (Hg.): Femina publica. Frauen, Öffentlichkeit, Feminismus, Köln: PapyRossa Verlag 1992. 


\section{Die Zeitschrift}

Im Materialkorpus befinden sich die zwei projekteigenen Zeitschriften der Milchjugend und des Jugendnetzwerks Lambda sowie in projektexternen Zeitschriften veröffentlichte Artikel der Villa und von TransInterQueer e.V. Die Zeitschriften und Artikel dienen sowohl der Herstellung einer internen Bewegungsidentität als auch einer alternativen Öffentlichkeit.

Die Zeitschrift, die als Trägermedium verschiedener Textsorten gilt, ist zwar ein altes, aber auch über soziale Bewegungen hinaus immer noch viel genutztes Medium. Im 17. Jahrhundert hießen Zeitschriften aus dem Französischen abgeleitet Journale. Erste Versuche, den Begriff ins Deutsche zu übersetzen, waren zunächst Bezeichnungen wie »Tagebuch«, »Monatsschrift«, »Wochenschrift«, »Sammlungen«, »periodische Schriften«, »Magazin« oder »Nachrichten«. Die Bezeichnung Zeitschrift ist Mitte des 18. Jahrhunderts zum ersten Mal verwendet worden und etablierte sich gegen Ende des 18. Jahrhunderts. ${ }^{9}$ Die ersten Zeitschriften sind im wissenschaftlichen Sektor erschienen, ${ }^{10}$ gefolgt von literarischen Rezensionszeitschriften und politisch-zeitgeschichtlichen Journalen. Mit der Aufklärungsbewegung sind, zunächst in England, erste sogenannte moralische Wochenzeitschriften erschienen. Später folgten Literaturzeitschriften, staatsbürgerliche Journale, Unterhaltungszeitschriften und, mit den neuen Druckmöglichkeiten, die Illustrierten. Erst als sich die Zensurbestimmungen auflockerten, wurden zu Beginn des 19. Jahrhunderts auch politisch-satirische Zeitschriften herausgegeben. Während im Nationalsozialismus Zeitschriften als wichtiges Medium des Propagandaapparates genutzt wurden, ist nach 1945 die Produktion von Zeitschriften durch die Alliierten gefördert worden. Diese erhofften sich von den Zeitschriften eine re-education durch Kulturaustausch. Aber auch Menschen aus dem Widerstand wollten mit der Gründung eigener Zeitschriften Demokratisierungsprozesse vorantreiben. ${ }^{11}$ Die erste Zeitschrift der Frauenbewegung erschien bereits 1849. Sie musste allerdings recht schnell wieder eingestellt werden. ${ }^{12}$

Damit blickt die Zeitschrift auf eine lange Geschichte zurück, in der sich deren Rolle je nach gesellschaftspolitischem System verändert und auch erweitert hat: Sie diente der Gleichschaltung durch Propaganda oder eröffnete Möglichkeiten, im demokratischen Sinne neue und alternative Wissensräume zu schaffen. Am Beispiel der Frauenbewegung zeigt sich, dass sie schon früh auch als Bewegungsmedium genutzt wurde. Mittlerweile hat sich eine Vielzahl unterschiedlichster Zeitschriften etabliert, die sich in Bezug auf Zielgruppe, Themen, Gestaltung, Sprache und Textsorten unterscheiden. Es gibt unter anderem Publikumszeitschriften, Fachzeitschriften, Special-Interest-Zeitschriften, Kunden- und Betriebszeitschriften, Verbands- und

9 Erich Straßner: Zeitschrift, Tübingen: Niemeyer Verlag 1997, S. 1.

10 Ebd., S. 4.

11 Erich Straßner: »Kommunikative Aufgaben und Leistungen der Zeitschrift«, in: Joachim-Felix Leonhard/Hans-Werner Ludwig/Erich Straßner et al. (Hg.), Medienwissenschaft. Ein Handbuch zur Entwicklung der Medien und Kommunikationsformen, New York: De Gruyter 1999, S. 852-864, hier S. 852-859.

12 Gisela Notz: »Der gefährliche Einfluss der Frauen-Blätter. Feministische Medienkultur in Deutschland«, in: Lea Susemichel (Hg.), Feministische Medien. Öffentlichkeiten jenseits des Malestream, Königstein/Taunus: Ulrike Helmer Verlag 2008, S. 29-40, hier S. 32. 
Vereinszeitschriften, Amtszeitschriften und Alternative Zeitschriften. ${ }^{13}$ Den unterschiedlichen Zeitschriften-Genres kommen auch verschiedene Aufgaben zu: Sie dienen der Unterhaltung, der Belehrung, der unterhaltenden Belehrung, dem Informationsaustausch, der Repräsentation nach außen wie dem Zusammenhalt und der Identitätsbildung nach innen. ${ }^{14}$ Je nach Zeitschriften-Genre variiert das Text-BildVerhältnis. Eine hohe Bildpräsenz in Zeitschriften fördert die affektive Vermittlung von Wissen und lässt die kurzen Texte in den Hintergrund treten. Charakteristisch für Zeitschriften ist deren relative Aktualität im Vergleich zu klassischen tagesaktuellen Nachrichten. Ziel ist es, tiefgehende und spezialisierte Berichte zu relativ aktuellen Geschehnissen $\mathrm{zu}$ verfassen. ${ }^{15}$ Die wichtigsten Textsorten, die im Trägermedium Zeitschrift versammelt sind, sind Glossen, Reportagen, Abhandlungen, Kommentare, Berichte, Briefe, Interviews, Zitate, kleine Feuilletons und poetische Werke. ${ }^{16}$ Die formale Gestaltung der meisten Zeitschriften ist standardisiert, um deren Unverwechselbarkeit zu gewährleisten.

Die zwei im Zentrum der vorliegenden Untersuchung stehenden Zeitschriften können als alternative Zeitschriften, die »Out!« auch als Verbandszeitschrift, eingeordnet werden. Auch ihre formale Gestaltung und das verwendete Textsortenspektrum entsprechen den genannten genrespezifischen Besonderheiten: Beide Zeitschriften verfügen über eine standardisierte formale Gestaltung und nutzen das für Zeitschriften charakteristische Textsortenspektrum von Gedichten über Interviews bis hin zu Berichten.

\section{Flyer und Broschüren}

Im Gegensatz zu den regelmäßig erscheinenden Zeitschriften sind Flyer, Broschüren und Plakate unregelmäßig erscheinende Medien, die zum Teil auch über mehrere Jahre hinweg verwendet werden können, weil sie kaum an Aktualität einbüßen. Flyer werden von allen fünf im Zentrum der vorliegenden Arbeit stehenden queeren Projekten hergestellt, um Veranstaltungen $\mathrm{zu}$ bewerben oder um über die eigenen Angebote $\mathrm{zu}$ informieren. Einige Flyer dienen auch der politischen Intervention. Mit Ausnahme der Milchjugend veröffentlichen ebenfalls alle Projekte eigene Broschüren. Die meisten Broschüren stellen Aufklärungs- und Informationsmaterialien zu bestimmten Themen für bestimme Zielgruppen bereit. Manche Broschüren informieren über das jeweilige Projekt und dessen Angebote.

Flyer und Broschüren gelten als Nachfolger der Flugschriften und Flugblätter. Diese Medien sind noch älter als die Zeitschrift und werden jenseits von sozialen Bewegungen und der Werbung kaum noch verwendet. Die ersten Flugblätter gab es bereits im 15./16. Jahrhundert. ${ }^{17}$ Die Bezeichnung Flugblatt, die sich ab dem 18. Jahrhundert durchsetzt, ist aus dem französischen feuille volante abgeleitet, was übersetzt fliegendes Blatt be-

\footnotetext{
13 E. Straßner: Zeitschrift, S. 25.

14 Ebd., S. 26-32.

15 E. Straßner: Kommunikative Aufgaben und Leistungen der Zeitschrift, S. 856.

16 E. Straßner: Zeitschrift, S. $18 \mathrm{ff}$.

17 Zu Beginn waren Flugblätter noch einfache Holzschnitte.
} 
deutet. ${ }^{18}$ In den Medienwissenschaften werden Flugschriften und Flugblätter wie folgt definiert:

»[U]nter >Flugblatt $<$ und >Flugschrift $<$ werden Druckerzeugnisse verstanden, die drucktechnisch aus einem Blatt bestehen, auch wenn sie beidseitig bedruckt sind (Fb.), bzw. aus ungebundenen und ohne Deckel versehenen mehreren Blättern (Fs.), deren gemeinsame Funktion es ist, zu aktuellen und umstrittenen Themen des Cemeinwohls Stellung zu nehmen, Meinungen zu formen und eventuell zu Handlungen aufzurufen. $\ll^{19}$

In der frühen Neuzeit hatten Flugblätter noch nicht so sehr die Funktion der Auflehnung und des Widerstandes, sondern eher eine »sozialdisziplinierende Funktion « ${ }^{20} \mathrm{Zu}$ Reformationszeiten nutzten theologische Institutionen Flugblätter und Flugschriften auch, um ihre Streitigkeiten öffentlich auszutragen. Im Deutsch-Französischen Krieg 1870/71 wurden Flugblätter dann erstmalig zur politischen Meinungsbildung verwendet. $^{21}$

Mittlerweile werden Flyer, neben der Werbung, vor allem in widerständigen politischen Zusammenhängen genutzt. In diesen Kontexten »hat das Flugblatt bis heute eine wichtige Funktion als unaufwendiges, schnell zu produzierendes und zu distribuierendes Medium «. ${ }^{22}$ Während bei Zeitschriften je nach Genre das Text-Bild-Verhältnis variiert, zeichnen sich Flugblätter zumeist durch ein ausgewogenes Text- und BildVerhältnis aus. Oft ist das Bild sogar dominant. Entsprechend werden sie als ein zweikanaliges Kommunikationssystem verstanden, in dem Text und Bild in einem komplementären Verhältnis zueinander stehen. Das Bild ist nicht identisch mit dem Sprachlichen, der Text hat aber häufig die Funktion, die Mehrdeutigkeit des Bildes in eine bestimmte Richtung zu lenken. ${ }^{23}$ Zentrale sprachlich-rhetorische Mittel von Flugblättern und Flugschriften sind Wortspiele, Metaphern, Metonymien, Schimpfwörter und Fahnenwörter. ${ }^{24}$ Die wichtigsten Textfunktionen der beiden "Mikromedien ${ }^{25}$ sind die aufklärende und belehrende Funktion sowie die Werbung. Bei Flugschriften dominiert im Gegensatz zum Flugblatt der geschriebene Text. Oft kommen auch gar keine Bilder zum Einsatz. Flugschriften bestehen meistens aus mehreren Blättern, die lose zusam-

Wolfgang Adam: »Theorien des Flugblatts und der Flugschrift«, in: Joachim-Felix Leonhard/HansWerner Ludwig/Erich Straßner et al. (Hg.), Medienwissenschaft. Ein Handbuch zur Entwicklung der Medien und Kommunikationsformen, New York: De Gruyter 1999, S. 132-143, hier S. 133. Joachim-Felix Leonhard/Hans-Werner Ludwig/Erich Straßner et al. (Hg.), Medienwissenschaft. Ein Handbuch zur Entwicklung der Medien und Kommunikationsformen, New York: De Gruyter 1999, hier S. 802.

20 W. Adam: Theorien des Flugblatts und der Flugschrift, S. 138.

21 J. Schwitalla: Flugblatt und Flugschrift II: Kommunikative und ästhetische Analysen, S. 810.

22 Dieter Burdorf/Christoph Fasbender/Burkhard Moennighoff: Metzler Lexikon Literatur. Begriffe und Definitionen, Stuttgart Weimar: Verlag J.B. Metzler 2007, S. $245 f$. 
mengeheftet sind. Entsprechend gelten diese auch als die Vorgänger von Broschüren, ${ }^{26}$ während Flyer als moderne Form des Flugblattes gelten.

Die Flyer und Broschüren der fünf im Zentrum der vorliegenden Untersuchung stehenden queeren Projekte werden in ihren genrespezifischen Funktionen genutzt. Sie dienen der Aufklärung, Informationsweitergabe und Veranstaltungsbewerbung. Je nach Funktion sind sie allerdings sehr unterschiedlich gestaltet und entsprechen nicht durchwegs den genrespezifischen Besonderheiten des Flugblatts. Einige Flyer - vor allem diejenigen, die der politischen Intervention oder Veranstaltungsbewerbung dienen - arbeiten mit einem ausgewogenen Text-Bild-Verhältnis, mitunter kommt dem Bild auch eine zentrale Rolle zu. Flyer mit informierender Funktion ähneln hingegen oft eher der Flugschrift. Hier dominiert der Text und es werden wenige, zum Teil auch gar keine Bilder verwendet. Auch die genannten gängigen sprachlich-rhetorischen Mittel kommen dort kaum vor. Bei den Broschüren der queeren Einrichtungen überwiegt, ähnlich wie bei den Flugblättern, der Text. Bilder und Illustrationen werden seltener genutzt und haben dann eher eine unterstützende Funktion.

\section{Das Plakat}

Einige der von den untersuchten Projekten veröffentlichten Flyer werden gleichzeitig auch als Plakate gedruckt. Hinzu kommen gezielte Plakatkampagnen von LesMigraS und dem Jugendnetzwerk Lambda BB sowie der Villa. ${ }^{27}$

Flugblätter und Flugschriften gelten als Vorläufer des Plakats. Im Vergleich zum Plakat wird Flugblättern allerdings eine mangelnde Ästhetik zugeschrieben. ${ }^{28}$ Die Wortherkunft geht vermutlich auf den im 16. Jahrhundert verwendeten niederdeutschholländischen Begriff plakaat zurück. Die lateinische Übersetzung des Wortes placare - »laut schreien« - und des Wortes plaga - »Blatt oder Fläche« - legt eine begriffliche Herkunft aus dem Lateinischen nahe. ${ }^{29}$ Das moderne Plakat wird folgendermaßen definiert:

»Das moderne Plakat ist ein auf stärkste optische Wirksamkeit ausgerichtetes grafisches Medium persuasiven Charakters, das durch seinen spezifischen Zweck (Werbung), eine bestimmte Materialbeschaffenheit (in der Regel Papier), ein gewisses Format (in Deutschland nicht kleiner als DIN A 3), eine spezielle Technik (in der Regel Druck nach Vorentwurf) und eine eigene Verbreitungsform (Aushang, Klebung an Säulen und Wänden) in seiner Identität bestimmt ist. ॥ $^{30}$

Helmut Schanze/Susanne Pütz: Metzler Lexikon Medientheorie und Medienwissenschaft. Ansätze - Personen - Crundbegriffe, Stuttgart Weimar: Verlag J.B. Metzler 2002, S. 40.

27 Hier sind die Plakate der PPÖ gemeint, die mit in den Materialkorpus aufgenommen wurden, weil viele Villa-Aktivist*innen daran beteiligt waren.

28 Dieter Fuder: »Kommunikative und ästhetische Funktionen des Plakats in ihrer geschichtlichen Entwicklung«, in: Joachim-Felix Leonhard/Hans-Werner Ludwig/Erich Straßner et al. (Hg.), Medienwissenschaft. Ein Handbuch zur Entwicklung der Medien und Kommunikationsformen, New York: De Gruyter 1999, S. 985-1001, hier S. 1001.

29 Johannes Kamps: »Theorien des Plakats«, in: Joachim-Felix Leonhard/Hans-Werner Ludwig/Erich Straßner et al. (Hg.), Medienwissenschaft. Ein Handbuch zur Entwicklung der Medien und Kommunikationsformen, New York: De Gruyter 1999, S. 148-161, hier S. 148. 
Die ersten Formen des Plakates entstanden in der frühen Neuzeit. Mit der Französischen Revolution wurde das Plakat erstmals als Mittel der politischen Meinungsbildung genutzt und in den beiden Weltkriegen diente es Propagandazwecken. ${ }^{31}$

Mittlerweile werden drei Plakatgattungen unterschieden: Werbeplakate, Filmplakate und politische Plakate. ${ }^{32}$ Mit der Professionalisierung der Waren- und Werbeplakate verloren die künstlerischen Aspekte der Plakatgestaltung zu Gunsten der Aufmerksamkeitsökonomie an Relevanz. ${ }^{33}$ Spätestens mit den 68er-Protestbewegungen hat das Plakat jenseits der Werbung wieder an Relevanz gewonnen und wurde »Zu einem wesentlichen Ausdrucksmittel der alternativen Bewegungen «. ${ }^{34}$ In sozialen Bewegungen steht oft, aufgrund mangelnder technischer Möglichkeiten, die Klarheit und Prägnanz im Vordergrund der Plakatgestaltung. Neben der politischen Intervention hat das Plakat dort auch eine maßgeblich identitätsstiftende Wirkung. ${ }^{35}$ Plakaten wird aufgrund der hohen Bildpräsenz eher eine gefühlsbetonte anstatt einer argumentierenden Wirkung zugeschrieben. Es gibt aber auch sachlich informierende Plakate. Generell gilt für Plakate, dass sie innerhalb von kurzer Zeit Aufmerksamkeit erregen und eine Botschaft vermitteln müssen und daher auf das Notwendige reduziert und leicht verständlich sein sollten. So funktionieren Plakate häufig über Vereinfachungen, Wiederholungen, Kontraste oder Symbole. ${ }^{36}$ Als zentrale sprachlich-rhetorische Strategien gelten die »enthymematische Schlußlogik«, der »disjunktive Stil« und »Metonymien «.37

Die in dieser Arbeit analysierten Flyer und Plakate haben, was Funktion, Umfang und Anordnung betrifft, sowohl Ähnlichkeiten mit dem Flugblatt als auch mit dem Plakat. Eine eindeutige Grenzziehung und damit Zuordnung ist nicht möglich. Je nach Budget und Ressourcen der Projekte sind diese Medien mal mehr und mal weniger aufwendig und entsprechend ästhetisch gestaltet. Die Plakate der Projekte werden für Aufklärungs- oder Sensibilisierungskampagnen verwendet, sie irritieren die politische Meinungsbildung im öffentlichen Raum, stellen Identifikationsangebote dar oder bewerben eigene Veranstaltungen. Mit Ausnahme der Veranstaltungsbewerbungen finden sich dort oft die für das Plakat genrespezifischen Eigenschaften wie beispielsweise Vereinfachungen oder Kontrastierungen wieder. wig/Erich Straßner et al. (Hg.), Medienwissenschaft. Ein Handbuch zur Entwicklung der Medien und Kommunikationsformen, New York: De Gruyter 1999, S. 1011-1016, hier S. 1011-1014.

32 Ebd., S. 1014.

33 J. Kamps: Theorien des Plakats, S. 151.

34 B. Denscher: Ceschichte des Plakats, S. 1015.

35 Protestplakate fanden sich beispielsweise in privaten Räumen.

36 J. Kamps: Theorien des Plakats, S. $151 \mathrm{ff}$.

37 Dieter Fuder: »Kommunikative und ästhetische Leistungen der Sprache im Plakat in ihrer geschichtlichen Entwicklung«, in: Joachim-Felix Leonhard/Hans-Werner Ludwig/Erich Straßner et al. (Hg.), Medienwissenschaft. Ein Handbuch zur Entwicklung der Medien und Kommunikationsformen, New York: De Gruyter 1999, S. 1001-1011, hier S. 1010. 


\section{Die Website}

Alle fünf der im Zentrum der vorliegenden Untersuchung stehenden Projekte verfügen über einen eigenen Homepageauftritt. Dieser bietet ihnen die Möglichkeit, relativ kostengünstig eine große Reichweite zu erlangen.

Der Homepageauftritt gilt als eines der ältesten Instrumente der OnlineKommunikation. ${ }^{38}$ Im Fall von Non-Profit-Organisationen sind zumeist die eigene Zielgruppe und die sogenannten Stakeholder Hauptbezugsgruppe der OnlineÖffentlichkeitsarbeit. ${ }^{39}$ Websites haben das Ziel, eine Art Selbstbild an die Öffentlichkeit zu tragen. Sie verfügen zudem häufig über Sub-Kommunikationsformen wie Live-Ticker, interaktive Angebote oder Foren. ${ }^{40}$ Es wird zwischen Websites, die als Plattform fungieren, Nachrichten-Websites und imageorientierten Websites unterschieden. Organisationen, Vereine und Verbände verfügen zumeist über eine imageorientierte Website. Diese besteht aus einer Startseite mit Begrüßung sowie aus Texten zu den Interessen, Zielen, Leitbildern und Tätigkeiten der Organisation. Viele solcher Websites besitzen auch eine eigene Presse-Ecke beziehungsweise Orte, an denen Material für die Presse - im Falle von Nicht-Regierungsorganisationen auch für die Zielgruppe und andere Gruppen - bereitgestellt wird. ${ }^{41}$ Im Gegensatz zu einem elektronischen Text, der linear organisiert ist, sind Websites durch Hypertextualität gekennzeichnet. Diese äußert sich vor allem in den dynamischen Verknüpfungen, die über Verlinkungen hergestellt werden. ${ }^{42}$ Des Weiteren ist die Entgrenzung von Websites gekennzeichnet durch multimodale Zeichenhandlungen. Das bedeutet, die Zeichen dienen der inhaltlichen Kommunikation, der Oberflächenstrukturierung und als Navigationsinstrument. ${ }^{43}$

Die Homepages der fünf queeren Projekte können als imageorientierte Websites eingeordnet werden. Die Einrichtungen informieren auf der Homepage über ihre Ziele, Aktionen, Veranstaltungen und Beratungsmöglichkeiten. Fast alle projekteigenen Homepages besitzen eine »Presse-Ecke«. Dort sind politische Stellungnahmen, Pressemitteilungen, Redebeiträge, aber auch viele der Print-Broschüren, Flyer und Plakate downloadbar und es werden Artikel und Materialien veröffentlicht.

\section{Pressemitteilungen, politische Stellungnahmen und Redebeiträge}

Mit dem Homepageauftritt ergibt sich auch die Möglichkeit, Pressemitteilungen, Stellungnahmen und Redebeiträge zu veröffentlichen. Dadurch kann die Reichweite dieser Public Relations: Wissenschaftliche Grundlagen und berufliches Handeln. Mit Lexikon, Wiesbaden: Springer Verlag 2015, S. 1017-1038, hier S. 1027. (Hg.), Handbuch der Public Relations. Wissenschaftliche Grundlagen und berufliches Handeln Mit Lexikon, Wiesbaden: Springer Verlag 2015, S. 631-649, hier S. 637. (Hg.), Handbuch Sprache im multimodalen Kontext, Berlin, Boston: De Gruyter 2016, S. 410-436, hier S. 413. 
Texte zeitlich und räumlich erweitert werden. Diese Möglichkeiten nutzen mit Ausnahme der Milchjugend und der Villa alle Projekte. Oft sind die Pressemitteilungen, politischen Stellungnahmen und Redebeiträge als PDF- oder Word-Dokumente downloadbar, zum Teil sind sie auch einfach nur über die Website abrufbar.

Die Veröffentlichung von Pressemitteilungen und Stellungnahmen auf der Homepage ermöglicht es Non-Profit-Organisation kostengünstig eigene Gegenöffentlichkeiten herzustellen. ${ }^{44}$ Pressemitteilungen und Stellungnahmen erscheinen meistens unregelmäßig, oft reagieren sie auf für die eigenen Ziele relevante und aktuelle Ereignisse oder platzieren eigene wichtige Themen. Aufgrund der elektronischen Form sind diesen Textsorten einige Besonderheiten zu eigen: Das Layout der Pressemitteilung oder der Stellungnahme wird zumeist passend zur Website gestaltet. Auf das OnlineLeseverhalten abgestimmt sind die Texte oft kurz und übersichtlich strukturiert, und entsprechend der Suchmaschinenoptimierung ist die Sprache an die Keyword-Sprache angepasst. Häufig stellen sogenannte Teaser den Link zum Downloaden der Pressemitteilungen oder Stellungnahmen bereit, die zumeist schon die relevantesten Informationen beinhalten, da sie ein Entscheidungskriterium für das Weiterklicken sind. ${ }^{45}$ Online-Pressemitteilungen werden im Kontext von Unternehmen folgendermaßen definiert:

»Eine auf der Homepage eines Unternehmens veröffentlichte Pressemitteilung stellt einen kohäsiven und kohärenten E-Text dar, der eine unternehmensbezogene Neuigkeit beschreibt und in die Textwelt des Unternehmens einordnet. Das Unternehmen verfolgt hierbei die Intention der Selbstdarstellung, während der Rezipient, meist ein Journalist, den Text zuerst als Informationsquelle sieht. $^{46}$

Die gängigsten Textsorten der Pressemitteilung sind Meldungen, Nachrichten und Berichte. Als für Pressemitteilungen ungeeignete Textsorten gelten Interviews, Reportagen, Kommentare und Glossen, wobei letztere sich für Stellungnahmen eignen. ${ }^{47}$

Stellungnahmen werden insofern von Pressemitteilungen unterschieden, als es sich bei ihnen um wertende Darstellungen zu politischen oder gesellschaftlichen Zuständen handelt. Sie stellen häufig eine Reaktion auf politische Entscheidungen/Ereignisse dar und sind umfangreicher ausgearbeitet als Pressemitteilungen. Oft liegt ihnen eine hohe fachspezifische Expertise zugrunde. ${ }^{48}$ In der Politolinguistik werden Stellungnahmen unter dem Oberbegriff der »Protestresolution« zusammengefasst. Ihre Aufgabe ist es, sich zu einem als »negativ erachteten Sachverhalt, der im tatsächlichen oder vermeintlichen Verantwortungs- und primären Adressatenbereich liegt«, zu positionie-

44 Katrin Bischl: Die professionelle Pressemitteilung. Ein Leitfaden für Unternehmen, Institutionen, Verbände und Vereine, Wiesbaden: Springer Verlag 2011, S. 161.

45 Ebd., S. $126 \mathrm{ff}$.

46 Ferdinand Wedler: Pressemitteilungen deutscher und französischer Unternehmen im Internet. Eine empirisch-linguistische Analyse, Stuttgart: ibidem-Verlag 2006, S. 53.

47 K. Bischl: Die professionelle Pressemitteilung, S. 81-94.

48 Vgl. Alexandra Seibt: Lobbying für erneuerbare Energien. Das Public-Affairs-Management von Wirtschaftsverbänden während der Gesetzgebung, Wiesbaden: Springer Verlag 2015. 
ren. ${ }^{49}$ Stellungnahmen können aus politischen Forderungen, Handlungsaufrufen oder Solidaritätsbekundungen bestehen oder die Durchsetzung bestimmter politischer Ziele hinterfragen. Sie beinhalten meistens eine Darstellung des Gegenstandes sowie eigene Forderungen und Begründungen. ${ }^{50}$

Eine weitere Textsorte, die zumeist online im Presse- oder Archivbereich der Projekte veröffentlicht wird, ist der Redebeitrag. Es handelt sich dabei zumeist um Reden, die auf Veranstaltungen oder Demonstrationen gehalten wurden. Im parteipolitischen Kontext wird Bezug nehmend auf Aristoteles zwischen politischen Reden, in denen Dissens und das Selbst als Teil der Auseinandersetzung im Vordergrund stehen, und Festreden beziehungsweise epideiktischen Reden, die eher konsensorientiert sind, unterschieden. ${ }^{51}$

Die genannten Unterscheidungen zwischen Pressemitteilungen und Stellungnahmen spielen in der Benennung der Textsorte durch die Projekte selbst keine Rolle. Die Einrichtung verwenden die Bezeichnungen »Pressemitteillungen« und »Stellungnahmen«, mitunter auch »Redebeiträge« weitgehend synonym. Manchmal fallen die Stellungnahmen etwas länger aus als die Pressemitteilungen. Gemeinsam ist den beiden Textsorten, wie sie von Lambda BB, TrIQ und LesMigraS verwendet werden, dass sie formal standardisiert und graphisch an die Homepage oder das Logo des jeweiligen Projekts angepasst sind. In den Überschriften wird die wichtigste Message vermittelt und beide Textsorten sind jeweils in kleine übersichtliche Absätze unterteilt. Die meisten Pressemitteilungen und Stellungnahmen sind nur ein bis zwei Seiten lang. Im Falle der von den Projekten veröffentlichten Reden handelt es sich oft um eine Mischung aus beidem (Politische Rede und Festrede). Demonstrationen und Veranstaltungen werden zum Teil gleichzeitig für Dankes-, Fest- und politische Reden genutzt. Es wird im Dissens mit der Community oder der Gesellschaft Kritik geübt, die eigene Arbeit hervorgehoben, es werden Forderungen gestellt, es wird aber auch konsensorientiert die Relevanz der Demonstration oder Veranstaltung betont.

Der Zugang zu massenmedialen Ressourcen hat sich mit dem Internet verändert. Vor allem eigene Websites und die Möglichkeiten, dort Stellungnahmen, Redebeiträge und Informationsmaterialien zu veröffentlichen, sind ein wichtiger Bestandteil der Öffentlichkeitsarbeit von politischen Einrichtungen geworden. Trotz alledem greifen alle der im Zentrum der vorliegenden Untersuchung stehenden queeren Projekte nach wie vor auch auf die schon seit Jahrhunderten existierenden Mediensorten wie Zeitschriften, Broschüren, Flyer und Plakate zurück. Während die dort verwendeten Trägermedien und Textsorten in den verschiedenen wissenschaftlichen Disziplinen wenig beachtet werden, spielen sie für soziale Bewegungen nach wie vor eine zentrale Rolle. Historisch betrachtet waren der Kostenpunkt und die Reichweite stets wichtige Gründe, auf diese Medien zurückzugreifen. Mit den Möglichkeiten der Online-Kommunikation gibt

49 Josef Klein: Crundlagen der Politolinguistik. Ausgewählte Aufsätze, Berlin: Frank \& Timme 2014, S. $192 f$.

50 Vgl. A. Seibt: Lobbying für erneuerbare Energien.

51 J. Klein: Grundlagen der Politolinguistik, S. 183f. 
es eine kostengünstige Alternative. Doch bringt diese auch einige Hürden und Nachteile für soziale Bewegungen mit sich. Zum einen überschreiten die Anforderungen nach Regelmäßigkeit und Aktualität in Online-Öffentlichkeiten wie Blogs oder sozialen Medien vermutlich die personellen Ressourcen der im Zentrum der vorliegenden Untersuchung stehenden Einrichtungen. Zum anderen können strukturelle Begrenzungen von Online-Kommunikationen beispielsweise durch Algorithmen, ökonomische Anordnungen oder technische Vorgaben, ${ }^{52}$ denen sich die Projekte vielleicht nicht unterwerfen wollen, eine Rolle spielen. Die Online-Kommunikation, die neben den sehr alten Mediensorten von den untersuchten Einrichtungen genutzt wird, unterliegt im Vergleich zu den Anforderungen an einen "guten« Social-Media-Auftritt weniger den Anforderungen nach Aktualität. Die Verwendung alter Mediensorten (Flyer, Plakate, Broschüren, Zeitschriften) und vergleichsweise älterer Möglichkeiten der Online-Kommunikation (Homepageauftritt, Pressemitteilungen, Stellungnahmen) ermöglichen zum einen im Vorfeld der Veröffentlichung eine gemeinsame Position auszuhandeln zum anderen eine tiefergehende Argumentation oder Auseinandersetzung im Text selbst.

\subsection{Die Beschreibung des Materialkorpus: Erste Einblicke}

Die Beschreibung der projektspezifischen, bewegungsinternen Materialien basiert auf Erkenntnissen der Strukturanalyse, die, im Sinne Jägers, der Auswahl repräsentativer Diskursfragmente vorausgeht..$^{53}$ Sie soll einen Einblick in und Überblick über das strukturanalytisch betrachtete Material geben und stellt methodisch gesehen zugleich einen Aspekt der Feinanalyse dar - nämlich die Darstellung des unmittelbaren institutionellen Rahmens, der in der Feinanalyse untersuchten Diskursfragmente. ${ }^{54}$ Hierzu werden die von den jeweiligen Einrichtungen verwendeten Medien, deren zentrale Inhalte, Funktionen und Zielgruppen sowie - insbesondere im Fall von Pressemitteilungen, Stellungnahmen und Redebeiträgen - die Anlässe der Veröffentlichungen beschrieben. Dadurch soll zum einen die Feinanalyse informiert werden; zum anderen soll dieser Teil auch einen Überblick über das gesamte Material geben, um Aspekte der Öffentlichkeiten der einzelnen Einrichtungen, die im Analyseteil nicht vorkommen, sichtbar zu machen. ${ }^{55}$ Nachdem die beiden bewegungsinternen Zeitschriften der zwei Jugendprojekte aufgrund der Regelmäßigkeit und Materialfülle einen großen Anteil des Materials ausmachen, werden diese etwas ausführlicher beschrieben.

Die Zeichenbegrenzungen in sozialen Medien verunmöglichen beispielsweise umfangreichere Argumentationen und setzen stark auf emotionale Kommunikation. H. Mitterhofer/M. Fritsche/T. Vogler/F. Madlung/M. Brandmayr: >UND WER SCHÜTZT UNS BÜRGER????<, S. 90.

53 S. Jäger: Kritische Diskursanalyse, S. 97.

54 Ebd., S. 99.

55 Für die Feinanalyse wurden Diskursfragmente zu Themen ausgewählt, die in allen fünf Projekten verhandelt werden. Andere Diskursfragmente, die zwar für die Öffentlichkeit der einzelnen Projekte, nicht aber für die Öffentlichkeiten aller Einrichtungen repräsentativ waren, wurden nicht für die feinanalytische Untersuchung ausgewählt. 


\subsubsection{LesMigraS-Öffentlichkeit: Gewalt und Rassismus}

Wichtige Instrumente der Antigewalt- und Antidiskriminierungsarbeit von LesMigraS sind Stellungnahmen, Pressemitteilungen, Redebeiträge, Online-Texte, Broschüren, Flyer und Plakate. Zwei Themen sind in der von LesMigraS hergestellten Öffentlichkeit zentral: zum einen das Thema Gewalt und Mehrfachdiskriminierung und der Umgang damit; und zum anderen Rassismus und Transphobie in der Community. Beide Aspekte werden thematisiert, indem die Einrichtung erstens sensibilisiert und aufklärt, zweitens Kritik äußert und drittens alternative Handlungsmöglichkeiten aufzeigt.

Die von LesMigraS veröffentlichten Broschüren sind alle im Rahmen von finanzierten Projekten entstanden und liegen in der eigenen Beratungsstelle aus. Sie sind aber auch online bestellbar und oft auch online downloadbar. Im Analysezeitraum von 2010 bis 2016 sind insgesamt fünf zum Teil sehr umfangreiche Broschüren veröffentlicht worden. Zwei Broschüren sind im Rahmen von »Tapesh ${ }^{56}$, eine im Rahmen des EUweiten Projekts »LARS ${ }^{57}$ und zwei im Zuge des Projektes »Language and Accessibility« entstanden. Gemeinsam ist den Broschüren der Fokus auf die beiden bereits genannten zentralen Themen von LesMigraS. Sie unterscheiden sich jedoch in Bezug auf die Zielgruppe und den Anlass der Herausgabe. Die Broschüre »Verbindungen sprechen ${ }^{58}$ ist $2011 \mathrm{im}$ Rahmen einer vom Projekt »Tapesh» organisierten Tagung erschienen, thematisiert Rassismus und Transphobie in der LBSTI-Community und diskutiert Möglichkeiten des Entkommens. Sie ist im A4-Format gedruckt und umfasst 47 Seiten mit Fachbeiträgen, Podiumsdiskussionen sowie Erkenntnissen aus den Workshops der Fachtagung. Die zweite der »Tapesh«-Broschüren, »Unterstützung geben «" sensibilisiert bezüglich Gewalt und Mehrfachdiskriminierung und eröffnet mögliche Handlungsoptionen im Umgang damit. ${ }^{60}$ Die Broschüre ist im A5-Format gedruckt und umfasst 67 Seiten. Weiters ist 2012 die etwas kleinere, aber inhaltlich ähnliche Broschüre "Handlungsmöglichkeiten bei Gewalt in Beziehungen ${ }^{61}$ erschienen. In dieser Broschüre liegt der Fokus speziell auf Gewalt in lesbisch/bisexuellen und Trans*Beziehungen. ${ }^{62}$ Zudem hat LesMigraS ganz im Sinne des eigenen intersektionalen Ansatzes zwei Broschüren in einfacher Sprache veröffentlicht. Beide Broschüren sind im A5-Format gedruckt und umfassen 22 beziehungsweise 23 Seiten. Sie sind 2012 und

»Tapesh« ist das Empowermentprojekt von LesMigraS, das in Kapitel vier näher beschrieben wird. »Lesbian awareness rising strategies to overcome the taboo of same-sex domestic violence«.

LesMigraS: Verbindungen sprechen.

LesMigraS: Unterstützung geben.

60 Die zwei Broschüren »Unterstützung geben« und »Verbindungen sprechen« sind durch Fördergelder von »Tapesh«, der »Initiative Berlin tritt ein für Selbstbestimmung und Akzeptanz sexueller Vielfalt« sowie dem Bundesprogramm »Toleranz fördern - Kompetenz stärken« des Bundesministeriums für Familie, Senioren, Frauen und Jugend gefördert. Ein zentraler Aspekt, der im Umgang mit Gewalt betont wird, ist die Unterstützung durch Freund*innen und Angehörige.

61 LesMigraS: Handlungsmöglichkeiten bei Gewalt in Beziehungen 2012.

62 Die etwas kleinere Broschüre »Handlungsmöglichkeiten bei Cewalt in Beziehungen - Eine Broschüre für lesbische/bisexuelle Frauen und Trans*-Menschen « ist gefördert im Rahmen des »Daphne III Programms« der EU sowie von dem Bundesministerium für Familie, Senioren, Frauen und Jugend und der Senatsverwaltung für Arbeit Integration und Frauen, der Landesstelle für Gleichbehandlung - gegen Diskriminierung. 
2013 erschienen, informieren leicht verständlich $\mathrm{zu}$ dem Beratungsangebot ${ }^{63}$ von LesMigraS und erklären Mehrfachdiskriminierung sowie Mehrfachbenachteiligung. ${ }^{64}$

Neben den Broschüren liegen in den Räumen von LesMigraS auch Flyer aus. Im Rahmen mancher Projekte gibt es explizite Flyer- und Plakatkampagnen, die größtenteils auch auf der Homepage downloadbar sind. So hat es im Zuge der Studie zu Mehrfachdiskriminierung 2012 eine Flyer- und Plakatkampagne mit dem Motto »Identität kennt kein Entweder-oder « gegeben. ${ }^{65}$ Ziel der Kampagne war es, für Mehrfachdiskriminierung zu sensibilisieren. Weitere Flyer aus dem Analysezeitraum machen, meistens in mehreren Sprachen, auf Gewalt gegen queere Personen aufmerksam oder fordern Solidarität und Selbstbestimmung. ${ }^{66}$ Die Flyer und Plakate sind meistens nur mit wenigen Wörtern, die durch Bilder oder Illustrationen unterstützt werden, beschriftet.

Zusätzlich zu den sowohl als Print als auch online zugänglichen Broschüren und Flyern veröffentlicht LesMigraS verschiedene Texte und Materialien, die nur über die Website des Projekts abrufbar sind.${ }^{67}$ Die Online-Texte sind in verschiedene Rubriken unterteilt, in denen LesMigraS über die eigenen Angebote aufklärt, das eigene Selbstund Gewaltverständnis expliziert, über Gewalt und Mehrfachdiskriminierung aufklärt, Leitfäden und Materialien herausgibt oder in die Community interveniert. Auch in den Online-Texten sind die beiden Themen - Rassismus und Transphobie in der Community sowie Gewalt und Mehrfachdiskriminierung - zentral: LesMigraS kritisiert beispielsweise Blackface-Praktiken, Pseudo-Einschlüsse sowie Ausschlüsse in der Community ${ }^{68}$ und veröffentlicht einen an die Community gerichteten Leitfaden für eine diskriminierungssensible Veranstaltungsorganisation. ${ }^{69}$ In den anderen Online-Texten wird Mehrfachdiskriminierung und Gewalt erklärt beziehungsweise auch das Gewaltverständnis $^{70}$ von LesMigraS expliziert. Zusätzlich stellt LesMigraS Materialien für den Umgang mit Gewalt wie beispielsweise einen Leitfaden zum Schreiben von Beschwerdebriefen und zur Erstattung von Anzeigen zur Verfügung.

Die Pressemitteilungen, Stellungnahmen und Redebeiträge von LesMigraS sind zumeist im PDF-Format auf der Homepage des Projekts in der Rubrik Archiv ${ }^{71}$ abrufbar. Aus dem Zeitraum 2010 bis 2016 sind insgesamt 29 Pressemitteilungen, Stellungnahmen und Redebeiträge in den Materialkorpus aufgenommen worden, die jeweils zwischen einer und zwei A4-Seiten umfassen. LesMigraS veröffentlicht Pressemitteilungen und Redebeiträge meistens zu queer-feministischen und antirassistischen Gedenk- und

63 LesMigraS: Informationen zum Beratungs-Angebot in leichter Sprache 2012.

64 LesMigraS: Wir erklären Mehrfach-Zugehörigkeit und Mehrfach-Benachteiligung. Text in leichter Sprache 2013.

65 LesMigraS: Studie, LesMigraS, https://lesmigras.de/studie_mehrfachdiskriminierung.html vom 27.08.2020.

66 LesMigraS: Archiv, https://lesmigras.de/Archiv.html vom 15.09.2020.

67 Es handelt sich um ca. 50 Seiten Online-Material.

68 LesMigraS: Antirassismus und LSBTIQ, https://lesmigras.de/antirassismus-und-Isbtiq.html vom 15.09.2020.

69 LesMigraS: Diskriminierungssensibilität, https://lesmigras.de/diskriminierungssensibilitaet.html vom 15.09.2020. 
Erinnerungstagen sowie Veranstaltungen. So publiziert die Einrichtung beispielsweise regelmäßig Pressemitteilungen zum Internationalen Tag gegen Homophobie, Transphobie und Interphobie (IDAHO_T_I), zum Transgender Day of Remembrance, zum Internationalen Tag gegen Rassismus und zum 8. März, oder spricht auf Veranstaltungen wie dem Transgenialen $\mathrm{CSD}^{72}$ in Berlin. In diesen Beiträgen positioniert sich LesMigraS zu aktuellen Debatten und thematisiert Rassismus, Sexismus, Homophobie, Trans*-Diskriminierungen und Diskriminierungen von Menschen mit Behinderungen in der LSBTIQ-Community und in der Gesellschaft. Zentrale Themen dieser Textsorten sind der CSD, Transphobie und Entpsychopathologisierung, Rassismus und Flucht sowie die Ausschlüsse in der Community.

\subsubsection{Die Türkis Rosa Lila Villa-Öffentlichkeit: Rassismus und Flucht}

Zentrale Medien einer gemeinsamen Villa-Öffentlichkeit sind die Banner an der Hausfassade, die Artikel in den »Lambda-Nachrichten«, die Flyer der Villa-Feste, die Jubiläums-Broschüren und der Villa-Blog. Andere mediale Öffentlichkeiten sind zum Teil eher einzelnen Gruppen wie den »Tipps« oder Aktivist*innen aus der Villa zuzuordnen. Im Analysezeitraum ist das zentrale Thema der Villa-Öffentlichkeiten Rassismus und Flucht. Das liegt nicht zuletzt auch an der Gründung des Vereins »Queer Base« im Jahr 2014. Bei den Veröffentlichungen der Villa zeigt sich, dass die Villa ein Projekt ist, das von vielen Menschen und verschiedenen Einrichtungen getragen wird. Entsprechend ist die Öffentlichkeit der Villa nicht einheitlich. Stattdessen werden viele verschiedene Themen auf unterschiedliche Art und Weise verhandelt.

Die bunte Hausfassade der Villa gilt bereits an sich schon als eine Intervention ins Wiener Stadtbild. Während die vielen Farben und Zeichnungen auf der Wand repräsentieren sollen, wer die Villa ist, stellen die an der Hauswand hängenden Banner eine zusätzliche Möglichkeit für öffentliche Statements dar. Die Reichweite dieser Statements geht oft über die Sichtbarkeit durch die Lage des Hauses hinaus. Viele - meistens kleinere lokale - Online- und Print-Medien berichten über die Positionierungen der Villa durch die Aufdrucke auf den Bannern, und auch die Villa selbst hat einen Blog auf der Homepage, auf dem oft die Statements auf der eigenen Hausfassade thematisiert werden. Meistens nutzt die Villa die Banner, um sich zu aktuellen national-, lokalund queer-politischen Themen zu positionieren. So solidarisiert sie sich beispielsweise mit der Belgrad-Pride, protestiert gegen die europäische Grenzschutzpolitik, gegen die FPÖ und ÖVP, gegen Rassismus oder ruft zur geschlechterpolitischen Solidarität auf. ${ }^{73}$ Kritik an der österreichischen Politik sowie das Thema Rassismus und Flucht sind im Analysezeitraum zentrale Themen der Bannerpolitik. Bei den Statements auf den Bannern handelt es sich um kurze Sätze oder kreative Wortspiele.

72 Der Transgeniale CSD ist ein alternativer CSD in Berlin.

73 Beispiele für Bannertexte: »Rosa Lila Villa gegen die Blau Schwarze Welle«, »Sichtbar und Selbstbewusst «, »wer* schweigt stimmt zu \#nohofer «, »Männerbünde sind unsexy « und »Fuck you WKR«, »Bleibe reicht für alle«, »Abartige gegen Abschiebung. Maria Frontex«, »Lesben sind keine Frauen und wollen auch keine werden«, »Lesben, Schwule und Transgender aller Galaxien vereinigt euch«. 
Ein weiterer Ort, an dem politische Statements, aber auch Berichte der Villa bis 2016 einen Raum hatten, sind die »Lambda-Nachrichten«. In der von der »Hosi Wien« herausgegebenen Zeitschrift veröffentlichte die Villa von 2014 bis 2016 regelmäßig Artikel in der Rubrik »Neues aus der Villa«. Die »Lambda-Nachrichten« liegen gratis in SzeneLokalen und Geschäften aus, sind online downloadbar und erscheinen seit 2010 fünfmal im Jahr. ${ }^{74}$ Sie bieten der Villa die Möglichkeit, eigene Themen in der Szene sichtbar zu machen und das Hausprojekt zu repräsentieren. In den im Analysezeitraum insgesamt 15 veröffentlichten Artikeln berichtet die Villa über aktuelle Projekte und Themenabende im eigenen Haus, diskutiert Ausschlüsse in der Villa oder thematisiert Rassismus. Es handelt sich meistens um kurze Berichte rund um die Villa und ihre Aktionen oder um Kommentare zur Villa-Politik oder zur Stadtpolitik. Meistens sind die Artikel eine oder zwei A4-Seiten lang. Besonders zentral ist das Thema Rassismus in diesen Artikeln.

Seit 2015 verfügt die Villa auch über einen eigenen Blog auf der Website, auf dem unregelmäßig Texte erscheinen. Der Villa-Blog besteht ebenfalls primär aus Berichten und Kommentaren und behandelt sehr unterschiedliche Themen, die zumeist die Villa oder lokalpolitische Debatten betreffen. Im Jahr 2015 sind mit fünf Einträgen die meisten getätigt worden, 2016 gibt es nur einen Eintrag. Auch hier ist, ähnlich wie in den »Lambda-Nachrichten«, das Thema Rassismus und Flucht zentral. Ein Aspekt des Blogs ist die mediale Verlängerung der Hausfassadenpolitik. So gibt es beispielsweise einen Eintrag zur »Tötet Schwule«-Hassbotschaft, die 2014 auf die Hauswand geschmiert wurde, oder einen Bericht, der den Aufdruck auf dem bis dahin größten Banner der Villa - mit dem Text »Abartige gegen Abschiebung« - als Aufhänger verwendet, um auf den neu gegründeten Verein »Queer Base« aufmerksam zu machen. ${ }^{75}$ Weiters wird, ähnlich wie auch in den »Lambda-Nachrichten«, über die Eröffnung von "Queer Base« berichtet, es wird aber auch aus aktuell gegebenem Anlass zum Beispiel Blackfacing in der Community kritisiert oder über das geplante »Denkmal für lesbischwule und Trans*gender Opfer des Nationalsozialismus in Wien « geschrieben. ${ }^{76}$

Die Villa produziert auch Flyer für die Villa-Feste oder Informationsflyer für die einzelnen Beratungseinrichtungen und Projekte, wie den »Türkis Rosa Tipp« und "Queer Base«. Alle fünf Jahre wird zudem eine Jubiläumsbroschüre ${ }^{77}$ veröffentlicht, in der über die Geschichte der Villa, die Beratungseinrichtungen, das Café, die Bildungsarbeit und vieles mehr berichtet wird. ${ }^{78}$ Darüber hinaus sind die einzelnen Beratungsstellen und Menschen zum Teil in nicht-Villa-übergreifenden Projekten aktiv, die auch in Veröffentlichungen münden. So gibt es beispielsweise das PPÖ-Projekt, an dem viele VillaMenschen beteiligt waren und dessen Flyer und Plakate im Archiv des »Türkis Rosa Tipps« zu finden sind. Die PPÖ, die Perverse Partei Österreichs, versucht, dem Rechtsruck in Österreich Humor und Ironie entgegenzusetzen. So hat die PPÖ für die Nationalratswahlen 2013 ihr eigenes "perverses« Wahlprogramm sowie Flyer und Plakatkam-

Hosi Wien: Lambda-Nachrichten, https://www.lambdanachrichten.at/ vom 15.09.2020.

Dieser Artikel wird auch feinanalytisch (im Ergebnisteil in Kapitel sieben) ausführlich dargestellt.

Türkis Rosa Lila Villa: Blog, https://dievilla.at/blog/ vom 16.09.2020.

Zumindest ist 2002, 2007 und 2012 eine Broschüre herausgegeben worden.

Türkis Rosa Lila Villa : 20 Jahre Rosa Lila Villa; Türkis Rosa Lila Villa : 15 Jahre Rosa Lila Villa 2007; Türkis Rosa Lila Villa : 30 Jahre Rosa Lila Villa 2012. 
pagnen erstellt. Auf ihren Wahlplakaten/Stickern und Postkarten kontrastieren sie Tradition, Heimat und Perversität. Auf den Plakaten und Flyern sind Männer im Dirndl, Frauen in Lederhosen und »Wahlslogans« wie »Wir legen die Wirtschaft in Fesseln«, »Peitschenhiebe und Heimatliebe« oder »Abartige gegen Abschiebung ${ }^{79}$ zu sehen.

\subsubsection{TransInterQueer e.V.-Öffentlichkeit: Entpathologisierung und Selbstbestimmung}

Die Einrichtung TrIQ gibt regelmäßig Statements oder Presserklärungen zu Trans*und Inter*-Themen $a b$, erstellt Broschüren, um bestimmte Ziel- und Berufsgruppen aufzuklären oder zu informieren, stellt Flyer bereit, um auf eigene Veranstaltungen oder Projekte aufmerksam zu machen, und schreibt vereinzelt Artikel. TrIQ interveniert vor allem in medizinische, juristische, mediale und Community-Diskurse, um für Trans* und Inter* zu sensibilisieren und um Entpsychopathologisierung, ein Ende normierender Eingriffe, trans* - und inter*-freundliche Berichterstattung und Unterstützung durch die Community zu fordern. Gemeinsamer Nenner der Öffentlichkeit von TrIQ ist das Ziel der Selbstbestimmung über den eigenen Körper.

TrIQ veröffentlicht im Analysezeitraum insgesamt 18 politische Stellungnahmen, Pressemitteilungen und Redebeiträge. Alle der in den Materialkorpus aufgenommenen Stellungnahmen, Pressemitteilungen und Redebeiträge sind auf der Homepage zu finden. Die Texte sind größtenteils als PDF-Datei downloadbar, einige sind nur als OnlineTexte verfügbar. ${ }^{80}$ Sie sind meistens ein bis zwei Seiten lang, manchmal gibt es aber sehr ausführliche Stellungnahmen, die bis zu 25 Seiten umfassen. Manche Stellungnahmen oder Pressemitteilungen werden in Kooperationen mit anderen Trans*- oder Inter*-Organisationen verfasst. Zentraler Gegenstand der beiden Textsorten sind gesetzliche und medizinische Richtlinien zu Trans* und Inter*. Dabei nutzt TrIQ häufig aktuelle Ereignisse auf lokaler, nationaler und internationaler Ebene, um Stellung zu beziehen. Auf nationaler Ebene werden beispielsweise Entscheidungen des Bundesverfassungsgerichts kommentiert und bundespolitische Entwürfe kritisiert; auch wird etwa das »Deutsche Institut für Menschenrechte« über die Situation von Trans* - und Inter*-Personen aufgeklärt. Auf internationaler Ebene werden unter anderem die Entscheidungen zu den Diagnosekriterien im ICD $^{81}$ oder DSM $^{82}$ kommentiert oder die $\mathrm{CEDAW}^{83}$ wird auf die verschiedenen Bedürfnisse von Trans*-Frauen aufmerksam gemacht. Neben den Interventionen in diese fachspezifischen Diskurse ist auch die Darstellung von Trans* in der medialen Berichterstattung zentraler Gegenstand der Pressemitteilungen. TrIQ solidarisiert sich aber auch mit einzelnen Trans*-Personen und

Perverse Partei Österreich: Die Perversen, https://www.facebook.com/DiePerversen/ vom 15.09.2020.

80 TransInterQueer e.V.: Politik \& Lobbyarbeit, https://www.transinterqueer.org/thema/politik-lobby arbeit/ vom 15.09.2020; TransInterQueer e.V.: Thema aktuell, https://www.transinterqueer.org/the ma/aktuell/ vom 15.09.2020. »Internationale statistische Klassifikation der Krankheiten und verwandter Cesundheitsprobleme«. »Convention on the Elimination of All Forms of Discrimination Against Women«. 
ihren Erfahrungen (z.B. mit einem schwangeren Trans*-Vater), interveniert, insbesondere auch mit Redebeiträgen, in die Community (z.B. den Transgenialen CSD, das Lesbisch-schwule Stadtfest) oder äußert sich zu lokalen Debatten (z.B. Unisex-Toiletten in Kreuzberg).

Im Untersuchungszeitraum hat TrIQ insgesamt acht Broschüren veröffentlicht, die alle auf der Homepage downloadbar sind. Sie sind meist sehr aufwendig und umfangreich, umfassen zwischen neun und 76 Seiten, im Durchschnitt sind es 25 Seiten. Entsprechend sind vor allem die umfangreicheren Broschüren auch finanziert ${ }^{84}$ und zum Teil in Kooperation mit anderen Einrichtungen und Organisationen entstanden. ${ }^{85}$ Die Zielgruppen der Broschüren sind medizinische Berufsgruppen sowie Trans*- und Inter*-Personen. In den drei Broschüren für medizinische Berufsgruppen - »Trans" ${ }^{*}{ }^{86}{ }$ Inter* $^{*}{ }^{87}$ und $»$ Intersektionale Beratung von/zu Trans* und Inter* ${ }^{88}$ - wird über trans*- und inter*-gerechte Sprache informiert, bezüglich normierender medizinischer Eingriffe sensibilisiert und über die negativen Aspekte von Diagnosen aufgeklärt. In der Broschüre »... And Others! ${ }^{89}$ werden Trans* - und Inter*-Personen selbst zu rechtlichen und medizinischen Aspekten informiert, sollten sie beispielsweise eine Personenstandsänderung oder eine Operation anstreben. Journalist*innen, die als wichtige Multiplikator*innen für Trans* - und Inter*-Belange gesehen werden, sind ebenfalls Zielgruppe der Broschüre "Trans* in den Medien ${ }^{90}{ }^{90}$ In dieser Broschüre wird über problematische Berichterstattung zu Trans*-Themen informiert. Zudem finden sich dort Hinweise, wie eine trans*-freundliche Berichterstattung aussehen

84 Die beiden Broschüren »Inter* und Sprache« und »Medizinische Eingriffe an Inter* und deren Folgen« sind im Zuge des von der »Senatsverwaltung für Arbeit, Integration und Frauen« sowie der »Landesstelle für Cleichberechtigung und gegen Diskriminierung «finanzierten »Inter* «-Projektes von TrlQ entstanden. Die Broschüre »... And Others!« ist finanziert durch das »Grundtvig Lifelong Learning Programm«der Europäischen Kommission. Die Broschüre »Intersektionale Beratung von/zu Trans* und Inter* « wurde durch das Förderprogramm »Offensive diskriminierungsfreie Cesellschaft «der »Antidiskriminierungsstelle des Bundes« gefördert.

85 So sind alle Inter*-Broschüren in Kooperation mit »Oll Cermany (Organisation Intersex International)«produziert worden.

86 TransInterQueer e.V.: Trans*. TrIQ informiert zum Thema Transgeschlechtlichkeit. Hinweise für Ärzt_innen, Psycholog_innen, Therapeut_innen und andere Berufsgruppen aus dem Cesundheitswesen 2013, https://www.transinterqueer.org/download/Publikationen/triq_infobroschuere_medi zinpsych_berufe.pdf vom 15.09.2020.

87 TransInterQueer e.V.: Inter*. Hinweise für Ärzt_innen, Psycholog_innen, Therapeut_innen \& andere medizinische Berufsgruppen 2013, https://www.transinterqueer.org/download/Publikationen/I nterSensibroschuere_2013.pdf vom 15.09.2020.

88 TransInterQueer e.V.: Intersektionale Beratung von/zu Trans* und Inter*. Ein Ratgeber zu Transgeschlechtlichkeit, Intergeschlechtlichkeit und Mehrfachdiskriminierung 2016, https://www.transin terqueer.org/wp-content/uploads/web_tis_brosch_aufl_3_161229.pdf vom 16.09.2002.

89 TransInterQueer e.V.: ... And Others! Argumentation Training for Transgender Inclusion in Europe. A >Good Practice Toolkit for Trans* Activists and Allies Working for Trans* Equality, Rights and Inclusion. 2011, https://www.transinterqueer.org/download/Publikationen/Transgender_Inclusion_A rgumentation_Toolkit.pdf vom 17.09.2020.

90 TransInterQueer e.V.: Trans* in den Medien. Informationen für Journalist_innen 2015, https://www .transinterqueer.org/download/Publikationen/TrIQ_Journalist_innen-2.\%20Aufl.-web(2).pdf vom 17.09.2020 
kann. Darüber hinaus gibt es Broschüren, die über die Lebenswelten, Erfahrungen und Bedürfnisse von Trans*- und Inter*-Personen informieren und an verschiedene Zielgruppen gerichtet sind. TrIQ hat eine Broschüre $\mathrm{zu}$ inter*-gerechter Sprache ${ }^{91}$ veröffentlicht, im »Trans* Inter* Queer $\mathrm{ABC}$ « $^{92}$ wird ein Überblick zu den wichtigsten Begrifflichkeiten gegeben und in einer weiteren Broschüre berichten Inter*-Personen über körperverletzende Erfahrungen durch medizinische Eingriffe. ${ }^{93}$ Grundsätzlich tragen die Broschüren zum einen dazu bei, zu Trans*- und Inter*-Themen $\mathrm{zu}$ informieren und $\mathrm{zu}$ sensibilisieren, zum anderen geben sie aber auch für bestimmte Zielgruppen konkrete Handlungsanweisungen für einen diskriminierungsärmeren und wertschätzenderen Umgang mit Trans*- und Inter*-Personen.

Neben den Broschüren und Stellungnahmen veröffentlicht TrIQ zudem Flyer und verfügt über einen eigenen Homepageauftritt. Die Flyer von TrIQ weisen zumeist auf das spezielle Angebot wie beispielsweise die Beratung, das Archiv oder eben das "Inter* «-Projekt hin. Auf der Homepage von TrIQ gibt es neben dem dort downloadbaren Material Informationen darüber, was TrIQ ist und macht. Hier handelt es sich vor allem um Selbstdarstellungen des Projektes, Positionierungen der Arbeit sowie Infos zu den Angeboten von TrIQ. Es wird beispielsweise erklärt, auf welcher Ebene Trans* und Inter* in einem Projekt vereint sind und was die Ziele von TrIQ sind. Seit 2016 schreibt TrIQ zudem im Rahmen des »Trans* Visible«-Projektes Artikel, die auf der Homepage des »Gunda-Werner-Instituts (GWI)« der »Heinrich-Böll-Stiftung« veröffentlicht werden. Die Artikel sind meistens ein bis zwei A4-Seiten lang und auf der Homepage des GWI in der Rubrik »LGBTI« zu finden. 2016 sind insgesamt vier Artikel veröffentlicht worden. In diesen Artikeln werden zumeist Erinnerungstage und Veranstaltungen zum Anlass genommen, um Trans*- und Inter*-Themen sichtbar zu machen.

\subsubsection{Jugendnetzwerk Lambda BB-Öffentlichkeit: Kampf um Räume und Coming-out}

Das Jugendnetzwerk Lambda BB nutzt vor allem Stellungnahmen und Pressemitteilungen für die eigene Öffentlichkeitsarbeit. Auf der Homepage finden sich zudem Selbstverständnisse und Informationen zu den eigenen Angeboten. Vereinzelte Plakat- und Flyer-Aktionen sind ebenfalls über die Website bestell- oder downloadbar. Zudem gibt es auf Bundesebene mit der »Out!« eine eigene Zeitschrift als Publikationsorgan. Drei Themen sind in der Öffentlichkeit des Jugendnetzwerks Lambda BB zentral. In den Pressemitteilungen, Stellungnahmen und Redebeiträgen ist das erstens die Forderung nach Räumen und finanziellen Ressourcen für die queere Jugendarbeit und zweitens die Auseinandersetzung mit Rassismus und Transphobie in der Community. In der »Out!«, genauso wie auf der Homepage, ist drittens das Coming-out das zentrale Thema.

Die Pressemitteilungen, Stellungnahmen und Redebeiträge von Lambda BB sind alle auf der Homepage im PDF-Format downloadbar und umfassen zum größten Teil ein bis

91 TransInterQueer e.V.: Inter* und Sprache.

92 TransInterQueer e.V.: Trans*Inter*Queer*ABC, https://www.transinterqueer.org/download/Publika tionen/TrIQ-ABC_web(2).pdf vom 20.11.2020.

TransInterQueer e.V.: Medizinische Eingriffe an Inter* und deren Folgen: Fakten \& Erfahrungen. 
zwei Seiten. ${ }^{94}$ Im Analysezeitraum sind insgesamt 31 Pressemitteilungen, Stellungnahmen und Redebeiträge veröffentlicht worden. Ein großer Teil dieser Textsorten dreht sich um die Forderungen nach mehr Finanzierungen und Räumlichkeiten für das Jugendnetzwerk $B B$, aber auch um bessere Strukturen in der queeren Jugendarbeit generell. So macht Lambda BB in den Pressemitteilungen immer wieder auf finanzielle Defizite aufmerksam, wenn die Einrichtung etwa davon berichten, politische Entscheidungsträger*innen an den »Runden Tisch« geladen zu haben, wenn sie die angedachten Kürzungen des »queer@school«-Projektes kritisieren oder wenn sie in einem Bedarfsantrag eine veränderte Regelfinanzierung fordern. Auch die mangelnden und nicht barrierefreien Räumlichkeiten werden zum Thema der Pressemitteilungen gemacht. Neben diesen Forderungen, die eigenen Ressourcen und Belange betreffend, informieren die Pressemitteilungen $\mathrm{zu} L a m b d a B B$-internen Veranstaltungen. Hier wird zumeist von eigenen Veranstaltungen und Projekten berichtet, wie zum Beispiel von dem Geburtstag des "In und Out«-Beratungsprojekts, der Wiedereröffnung vom »Lambda ${ }^{2}$ « oder dem Beginn der »LesBiSchwule Bus T*our«. Zusätzlich zu den Lambda BB selbst betreffenden Themen positioniert sich die Einrichtung auch zu aktuellen lokalpolitischen, aber auch darüber hinausgehenden queeren Debatten. So kritisieren sie beispielsweise die geringe Berücksichtigung von Trans* im Rahmen der »Bundesstiftung Magnus Hirschfeld« und kommentieren die Diskussion um den Bildungsplan in Baden-Württemberg oder die Unsichtbarmachung von bestimmten Gruppen, wie zum Beispiel Trans* of Color in der Verfilmung von »Stonewall«. Auf lokaler Ebene solidarisieren sie sich zum Beispiel mit der Nicht-Teilnahme von LesMigraS am Lesbisch-schwulen Stadtfest, bringen sich in die Diskussion zu Unisex-Toiletten in Kreuzberg ein oder sprechen am Berliner CSD. In diesen Positionierungen sind vor allem Mehrfachdiskriminierung und der Umgang damit Thema. ${ }^{95}$

Auf der projekteigenen Homepage informiert Lambda BB über die Angebote, ${ }^{96}$ die eigene Geschichte ${ }^{97}$ und die Einrichtung. Darüber hinaus sind Bildungsmaterialien downloadbar oder bestellbar. ${ }^{98}$ Das Coming-out ist das einzige Thema, zu dem es Informationen über die Selbstdarstellungen des Projektes hinaus gibt. Das »queer@school«Projekt verfügt über einen eigenen Homepageauftritt, auf den die Website des Jugendnetzwerks Lambda BB verlinkt. Neben den Informationen zu dem Projekt und den Angeboten positionieren sich die Jugendlichen des »queer@school«-Projekts in Bezug auf ihr Machtverständnis, Privilegien und Mehrfachdiskriminierung. ${ }^{99}$

\section{"Out! - Die Verbandszeitschrift des Jugendnetzwerks Lambda}

Die »Out!« ist seit 2007 die Verbandszeitschrift des Jugendnetzwerks Lambda. Sie ist die Nachfolgezeitschrift der »Lambdatio«, die bereits 1990 zum ersten Mal erschienen

Die längste Pressemitteilung umfasst 15 Seiten.

Jugendnetzwerk Lambda:BB: Pressemitteilungen.

Jugendnetzwerk Lambda:BB: Beratung, https://www.lambda-bb.de/beratung vom 20.08.2020; Jugendnetzwerk Lambda:BB: Jugendgruppen.

Jugendnetzwerk Lambda:BB: Unsere Geschichte.

Jugendnetzwerk Lambda:BB: Printmaterialien.

Jugendnetzwerk Lambda:BB: queer@school. 
ist. ${ }^{100}$ Die Bezeichnung »Out!« nimmt Bezug auf das Coming-out, um das bei Lambda nicht nur die Beratungsformate, sondern auch die Zeitschrift angeordnet ist. In der »Out!« schreiben queere Jugendliche füreinander. Die letzte Verantwortung für die Zeitschrift liegt allerdings beim Vorstand des Verbandes - also nicht bei den Jugendlichen. Die Zeitschrift erscheint seit Sommer 2007 alle drei Monate, also vierteljährlich. Sie war im Analysezeitraum kostenlos online zugänglich oder konnte auf Anfrage per Post zugesandt werden. Mitglieder und Fördermitglieder bekommen die Zeitschrift ebenfalls zugeschickt. Zwischen 2010 und 2016 hatte die Zeitschrift eine Auflage von 3300 bis 3500 Stück pro Ausgabe. Sie ist im A4-Format gedruckt und umfasst mit Ausnahme von Ausgabe 15 (28 Seiten) immer exakt 20 Seiten. Finanziell fördert der »Kinder- und Jugendplan des Bundes« die »Out!«, gedruckt wird die Zeitschrift im Eigenverlag. In den Jahren 2010 bis 2016 sind insgesamt 27 Ausgaben erschienen. ${ }^{101}$ Ähnlich wie in vielen primär ehrenamtlich organisierten aktivistischen Zeitschriften, ist die Fluktuationsrate in der Redaktion recht hoch. Die formale Gestaltung der Zeitschrift ist, wie es bei regelmäßig erscheinenden Zeitschriften zumeist der Fall ist, einheitlich. Das Deckblatt besteht aus dem Titelbild, dem Titel der Ausgabe sowie dem Logo des Jugendnetzwerks. Die Titelbilder der »Out!« stehen im Bezug zum Thema der jeweiligen Ausgabe. ${ }^{102}$ Es handelt sich mal um Zeichnungen, mal um Fotografien, mal um Graphiken. Auf dem Cover sind neben dem Titelbild der Name der Zeitschrift sowie die Nummer der Ausgabe, die Jahreszeit und das Erscheinungsjahr abgedruckt. Daneben steht etwas größer "Zeitschrift des Jugendnetzwerk Lambda e.V.«. Auf der Rückseitseite finden sich jeweils verschiedene Werbungen für anstehende Lambda-Veranstaltungen, oder Lambda versucht, Mitglieder zu mobilisieren. In der »Out!« überwiegt der Textanteil. Jeder Artikel ist aber mit einem Bild, einer Zeichnung oder einer Graphik unterlegt. Zumeist sind es thematisch passende Bilder oder Bilder von Personen. Wobei nicht immer erkenntlich ist, in welchem Bezug die abgebildeten Personen zum Artikel stehen.

Der inhaltliche Aufbau der Zeitschrift hat sich in den letzten Jahren bis auf ein paar kleine Variationen kaum verändert. Jede Ausgabe hat einen eigenen Themenschwerpunkt. ${ }^{103}$ Manche Themen beziehen sich auf Geschlecht und Sexualität wie beispielsweise »Queer«, »Trans« oder »Bisexualität«. Andere Themen wie »Mode«, »Sucht«, »Träume« oder »Internet« werden aus einer queeren Perspektive beleuchtet. Manchmal werden die Themenschwerpunkte auch anlässlich aktueller Ereignisse ausgewählt. Neben der Themen-Rubrik gibt es fünf weitere Rubriken, die in fast jeder Ausgabe zu finden sind: Mein Coming-out, Denkwürdiges, Rezension, Lambda intern

100 Jugendnetzwerk Lambda: Endlich Volljährig. Lambda wird 18, Out! 2008, S. 7.

101 Normalerweise werden pro Jahr vier Ausgeben, je eine im Frühling, Sommer, Herbst und Winter, veröffentlicht.

102 Meistens stammen die Bilder von Fotodatenbanken oder professionellen Fotograf*innen beziehungsweise Graphiker ${ }^{*}$ innen. Manche Titelbilder sind von privaten Personen, lizenzfrei oder von Mitarbeiter*innen der Zeitschrift.

103 In den Jahren 2010 bis 2016 sind folgende Themenschwerpunkte gesetzt worden: Familie, Sportlicher Sommer, Wohnen, Internet (2010); Geheimnisse, Trans, Internationalität, Geschichte (2011); Konsum, auf dem Land, Sucht (2012); Lügen, Glaube, Träume, Körper (2013); Bisexualität, Lust, Engagement, Medien (2014); Lernen, Flucht, Queer, Feiern (2015); Spiele, Mode, Sprache, Alter (2016). 
und die Kolumne. Auf der ersten Seite der Zeitschrift ist auf der rechten Seite das Editorial, Impressum und Inhaltsverzeichnis und auf der linken Seite ein Comic, meist mit Bezug zur aktuellen Ausgabe, abgedruckt. Im Editorial wird meistens auf vergangene und zukünftige Ereignisse und Veranstaltungen verwiesen und in das Thema der Zeitschrift eingeführt. Im Anschluss folgt die Rubrik »Denkwürdiges«, die im Frühjahr 2014 in "Que(e)rgeblickt « umbenannt wurde. In dieser Rubrik finden sich kurze Meldungen zu aktuellen »queeren« Themen, zum Beispiel dazu, welche prominente Person sich gerade geoutet hat oder wie die aktuelle Gesetzeslage zu Ehe und Lebenspartner*innenschaft in Deutschland aussieht. Im Anschluss daran folgen verschiedene Beiträge zu dem spezifischen Thema der jeweiligen Zeitschrift, es handelt sich dabei um Interviews, kurze Reportagen, Erfahrungsberichte, Berichte, Kommentare, Glossen und fiktive Geschichten. Ebenfalls in jeder Zeitschrift, der Ort variiert allerdings, gibt es eine Coming-out-Geschichte, die von queeren Jugendlichen eingesandt wurde. In jeder Ausgabe wird dazu aufgerufen, die eigene Geschichte zu erzählen: »Erzählt von eurem Coming-Out! Wir möchten wissen, wie ihr euer Coming-Out erlebt habt. War euch von Anfang an klar, dass ihr homo-, bi- oder transsexuell seid? Oder war es für euch schwer, diese Tatsache zu akzeptieren? Wie haben Freunde, Familie und Bekannte reagiert?! « ${ }^{104}$ Nach der Coming-out-Geschichte folgen meistens Buch- und Filmrezensionen. In einer weiteren Rubrik wird über Aktuelles aus dem Verband berichtet, wobei sich die Bezeichnung der Rubrik immer wieder ändert. ${ }^{105}$ Hier werden (Vorstands-)Mitglieder und Mitarbeiter*innen verabschiedet und vorgestellt, Veranstaltungen angekündigt beziehungsweise wird über vergangene Veranstaltungen/Netzwerktreffen berichtet. Es werden neue und alte Projekte vorgestellt, es gibt Informationen aus den Landesverbänden, Fortbildungsmaßnahmen und Mitgliederversammlungen werden beworben und Leiter*innen oder Unterstützer*innen für Veranstaltungen oder Projekte gesucht. Zudem gibt es sehr ausführliche Berichte zu den jeweiligen Lambda-Veranstaltungen wie beispielsweise den jährlich von Lambda organisierten Israel-Reisen und Sommercamps. Zum Schluss erscheint meistens eine Kolumne, die über einen längeren Zeitraum von denselben Autor*innen verfasst wird. So gab es im Analysezeitraum die »23 Minuten Kolumne von Oliver Spinedi«, in der dieser von Alltäglichem in seinem Leben berichtet, die Kolumne "Selbstgespräche im Zug« und später eine Glosse von Anna Bohlika. In den Kolumnen berichten die Autor*innen zumeist über eigene Erfahrungen oder erläutern eigene Gedanken. Oft wird ein Bezug zum Thema der Ausgabe hergestellt.

Die inhaltliche Ausrichtung der Zeitschrift wird vor allem in den Artikeln zu dem jeweiligen Thema der Zeitschrift, aber auch in der Rubrik »Denkwürdiges « und den Coming-out-Geschichten sichtbar. Die ganze Zeitschrift ist zunächst einmal grundlegend um das Coming-out herum angeordnet. Daneben sind der queere Aktivismus und die Darstellung geschlechtlicher und sexueller Vielfalt zentrale Themen der »Out!«. In fast jedem themenbezogenen Artikel ist das Coming-out Thema oder sogar zentral. Zusätzlich gibt es in jeder Ausgabe eine Coming-out-Geschichte. Meistens sind dies

104 Jugendnetzwerk Lambda: Träume, Out! 2013.

105 Folgende Bezeichnungen wurden im Analysezeitraum für die Rubrik verwendet: »Aus den Verbänden«, »Lambda intern« oder »Lambda informiert«, »Aus dem Verband«, »Infos aus dem Verband«. 
Geschichten über ein Coming-out, das nicht immer einfach, am Ende aber doch erfolgreich verlaufen ist. Darüber hinaus ist der aktuelle und bewegungsgeschichtliche queere Aktivismus ein zentrales Thema in der »Out!«. So werden verschiedene queere Gruppen oder Projekte vorgestellt, beispielsweise eine Selbsthilfegruppe für Eltern, queere Wohnungsprojekte, queere Senior*innen-Arbeit oder Beratungs- und Unterstützungsorte für bisexuelle Personen. Der queere Aktivismus wird aber auch bewegungsgeschichtlich kontextualisiert, wenn beispielsweise über die Schwulenbewegung berichtet, der LGBT-Aktivismus früher und heute verglichen oder von der Entstehung der Gender- und Queer-Studies erzählt wird. Neben dem Aktivismus spielt die Darstellung vielfältiger vergeschlechtlichter und sexueller Seinsweisen eine wichtige Rolle in der »Out!«. Es gibt Artikel zu Trans*, Inter*, Queer, über binationale Partnerschaften, $\mathrm{zu}$ queeren Menschen auf der Flucht, queeren Menschen mit Behinderung, Biund Pansexualität und vielem mehr. Es wird aber auch davon berichtet, wie die queere Community beispielsweise Bisexualität diskriminiert oder über Körperideale Menschen mit Handicap abwertet und ausschließt. Neben diesen drei zentralen Themen wird zudem häufig über Beziehungen und Partner*innensuche, beispielsweise über Online-Dating-Plattformen, geschrieben. Eine weitere Rubrik, die für die Ausrichtung der Zeitschrift repräsentativ ist, ist die Rubrik »Denkwürdiges«, später »Que(e)rgeblickt«. Sie ist so etwas wie ein queeres Kurznachrichtenportal. Die Rubrik umfasst eine Doppelseite und besteht meistens aus vier kurzen Berichten pro Seite, die jeweils mit einem Bild unterlegt sind. Auch hier ist der aktuelle queere Aktivismus ein zentrales Thema. So wird beispielsweise über die Insolvenz von queeren Veranstaltungen, den Tod von bekannten Aktivist*innen, Ausstellungen, Antidiskriminierungsaktionen, Auszeichnungen oder die »Transgender Awareness Week« berichtet. Besonders häufig sind aber auch Meldungen rund um die rechtliche Gleichstellung von Homosexualität. Es wird über Themen wie Adoptionsverbot, Ehe für alle, Gleichstellung der eingetragenen Partner*innenschaft mit der Ehe, selten auch über Trans*-Rechte und Asylrecht (jeweils einmal), informiert. Neben diesen Neuigkeiten, die Rechte zumeist homosexueller Menschen betreffend, wird ebenso häufig über Diskriminierung und Ausgrenzung von queeren Personen vor allem in Ländern des globalen Südens und des ehemaligen Ostblocks berichtet. Es gibt Meldungen zu Hinrichtungen, Gefängnisaufenthalten, CSD-Verboten, Hass-Verbrechen und homophoben politischen Entscheidungen oder Bemerkungen. ${ }^{106}$ Weitere zentrale Themen in dieser Rubrik sind das Coming-out, aber auch Eheschließungen von prominenten Personen.

Zusammenfassend kann die Verbandszeitschrift des Jugendnetzwerks Lambda als ein Ort des Austauschs für Jugendliche beschrieben werden, der zentral um das Comingout und den aktuellen queeren Aktivismus angeordnet ist und den Jugendlichen im Sprechen über die vielfältigen Möglichkeiten des geschlechtlichen und sexuellen Seins Identifikationsangebote bereitstellt. Damit ist die »Out!« gleichzeitig Bewegungszeitschrift und offener Austauschort, der Jugendlichen alternative Identifikationsangebote bereitstellt.

106 Demgegenüber wird in Bezug auf Länder im globalen Norden über die Legalisierung der HomoEhe, kirchliche Trauungen von Homosexuellen oder (pro-)homosexuellen Politikerinnen, über mehr Rechte für Trans*-Menschen oder Vielfalt in Schulbüchern berichtet. 


\subsubsection{Die Milchjugend-Öffentlichkeit: Stolz und widerständig}

Anders als in den anderen vier Projekten kommen Stellungnahmen und Flyer bei der Milchjugend nur sehr vereinzelt vor und auch der Homepageauftritt informiert hauptsächlich über die vielfältigen Veranstaltungen und Welten der Milchjugend. Stattdessen ist bei der Milchjugend die eigene Zeitschrift - das »Milchbüechli« - der Ort, an dem eine Bewegungsidentität sowie eine alternative Öffentlichkeit hergestellt werden. Das »Milchbüechli« oder auch die »falschsexuelle Zeitschrift der Milchjugend« ist die erste von mittlerweile neun Welten der Milchjugend. Herausgegeben wird die Zeitschrift vom »Verein queere Jugendplattform«, sprich der Milchjugend, gedruckt wird sie im AZ print Aarau. In den Jahren 2012 bis 2016 sind insgesamt 18 "Heftlis« herausgegeben worden. Im Gründungsjahr 2012 waren es zwei Ausgaben, danach jedes Jahr vier Ausgaben. Das »Milchbüechli« umfasst zumeist zwischen 20 und 30 Seiten im A4-Format. Die Zeitschrift ist gratis oder zu einem "Solipreis« von 2 Schweizer Franken erhältlich, ein Jahresabonnement kostet im Analysezeitraum 20 Schweizer Franken. Außer durch die Abos erreicht die Zeitschrift ihre Leser*innenschaft dadurch, dass sie in Infound Beratungsläden, in Buchhandlungen, Gemeinschaftszentren, der Aids-Hilfe, Einrichtungen der Jugendarbeit und verschiedenen Schulen ausliegt. Hauptzielgruppe der Zeitschrift sind queere Jugendliche in der Schweiz sowie Freund"innen und interessierte Jugendliche. Das Projekt wird vom »Bundesamt für Sozialversicherung« gefördert. Zwischen 2014 und 2015 war es auch Teil des europäischen Förderprogramms »Jugend in Aktion«. Zusätzlich wird das »Milchbüechli« von queeren beziehungsweise LGBTIQOrganisationen über das Konzept der Partner*innenschaft unterstützt; die Organisationen erhalten dafür Zeitschriften und werden als Freund"innen gelistet. Die Auflagenhöhe des »Milchbüechlis« schwankt zwischen 4000 und 6000 Stück pro Ausgabe. Laut eigener Statistik ist die Zahl der abonnierten Hefte in den letzten fünf Jahren von ca. 400 auf ca. 3400 gestiegen. ${ }^{107}$ Über Facebook-Veranstaltungen lädt die Milchjugend regelmäßig zu Redaktionssitzungen ein, an denen jede interessierte Person teilnehmen kann. Meistens gibt es über einen längeren Zeitraum Personen, die bestimmte redaktionelle Verantwortungsbereiche übernehmen und von anderen variierend unterstützt werden.

Bei der formalen Gestaltung des »Milchbüechli« fällt zunächst auf, dass es aus dünnem, Zeitungspapier-ähnlichem Papier besteht. Auf dem Cover sind meistens Einzel- oder Gruppenporträts von »falschsexuellen« Jugendlichen abgebildet. Analog zur Selbstpositionierung der Zeitschrift - „Denn wir sind nicht normal. Und das ist wunderbar. Das >Milchbüechli ist der Ort, um das zu zeigen ${ }^{108}$ - spiegeln die Porträts auf dem Cover die Vielfalt, aber auch den Stolz und das Selbstbewusstsein der Milchjugend wider. Neben den Porträts befindet sich auf dem Titelblatt der Name der Zeitschrift - »Milchbüechli. Zeitschrift für die falschsexuelle Jugend«-, der ab Juni 2016 in »Milchbüechli. Die falschsexuelle Zeitschrift der Milchjugend« geändert wurde. Zudem stehen die Nummer des Heftes, der Monat und das Jahr der Erscheinung sowie das Thema der jeweiligen Ausgabe auf dem Titelblatt. Auch auf der Rückseite

107 Milchjugend: Ausgabe 20.

108 Milchjugend: Wir machen Druck. 
der Zeitschrift sind, ähnlich wie auf dem Coverbild, Porträtfotos von »falschsexuellen« Jugendlichen zu sehen. Bis zur neunten Ausgabe wird das Bild auf der Rückseite der Zeitschrift durch einen Text unterlegt, der die Haltung der Milchjugend repräsentiert: »Wir kümmern uns nicht darum, was die anderen über uns sagen. Wir werden uns nie rechtfertigen. Wir wollen uns wehren. Sie nennen uns schwuchtelig, verkehrt oder pervers? Wir sind stolz darauf, so falsch zu sein. Auch du kannst stolz auf dich sein, so wie du bist. ${ }^{109}$ Später werden dann auch Veranstaltungen der Milchjugend, wie die »Molke 7-Party« oder das »Festival Lila«, auf der Rückseite beworben. Der inhaltliche Aufbau der Zeitschrift variiert im Vergleich zur »Out!« häufiger. Aber auch das "Milchbüechli« hat einige Rubriken, die in fast jeder Ausgabe vorkommen. Während in den ersten zwei Ausgaben auf den ersten zwei Seiten noch ein Inhaltsverzeichnis $\mathrm{zu}$ finden war, fällt dieses ab der dritten Ausgabe ganz weg. Stattdessen befindet sich in den nächsten Zeitschriften an dieser Stelle eine Liste von Orten, an denen das »Milchbüechli« ausgelegt wird. Ab der dritten Ausgabe werden auf den ersten zwei Seiten zudem die Partner*innen der Milchjugend aufgelistet. Zusätzlich gibt es auf den ersten zwei Seiten immer ein Impressum, in dem die an der Zeitung mitarbeitenden Jugendlichen und ihre Funktionen aufgelistet werden. Auf der zweiten Seite befindet sich das Editorial, dem ab der zweiten Ausgabe meistens auch ein Foto des aktuellen Redaktionsteams hinzugefügt ist. Im Editorial werden zumeist kurz die Themen der jeweiligen Ausgabe zusammengefasst. Im weiteren Aufbau der Zeitschrift gibt es einige wiederkehrende Rubriken, allerdings ohne festgeschriebene Reihenfolge. Eine Rubrik, die in fast jedem Heft vorkommt, ist das »Hilfe-Eggli«. Unter dem Motto »Was du schon immer wissen wolltest und dich nie getraut hast!« können Fragen gestellt werden. Ausgewählte Fragen werden dann in der Rubrik »Hilfe-Eggli« beantwortet. Zudem wird in der Fragestunden-Rubrik immer auch auf Notfallnummern, Notfallchats und Beratungseinrichtungen verwiesen. Eine weitere Rubrik, die in fast jeder Ausgabe zu finden ist, ist »Der Jugend erste Male«, in der die Jugendlichen von ihren ersten Küssen, zweifelhaften Hetero-Beziehungen, Sexshop-Besuchen, Sex-Partys, gemeinsamen Nächten, Outings und vielem mehr berichten. In fast jeder Ausgabe ist ein Kalender zu finden, in dem aktuelle Veranstaltungen sowie Jugend/Student"innenGruppen in der Schweiz und deren Treffen aufgelistet sind. In der Mitte des Hefts sind meistens Bilder von Veranstaltungen der Milchjugend, wie der »Milchreise und der "Jugendpride«, aber auch kreative Textsorten, wie beispielsweise ein Bullshitbingo, abgedruckt. Neben diesen regelmäßig erscheinenden Rubriken lebt die Zeitschrift von ihren vielseitigen Artikeln. Jede Ausgabe hat einen Themenschwerpunkt. Die Artikel nehmen allerdings mal deutlicher und mal weniger eindeutig Bezug auf das Thema der jeweiligen Ausgabe. ${ }^{110}$ Nach der 16. Ausgabe wurde auf einen Themenschwerpunkt verzichtet.

109 Milchjugend: Triff uns Live, Milchbüechli 2013.

110 In den Jahren 2012 bis 2016 sind folgende Themenschwerpunkte gesetzt worden. »Wir machen Druck«, »Oben Ohne« (2012); »Drei und mehr«, »Triff uns live!«, »An der Oberfläche«, »Crenzwerte« (2013); »Leiden_Schaft«, »Dein Freundschaftsbüechli«, »Wusstest du schon?«, »Hauptgang« (2014); »Worte der Macht«, »Grenzen überwinden«, »Für immer und ewig«, »Schlüsselerlebnis« (2015); »Falschsexuell die beste Phase meines Lebens!«, »Alles echt?«, »Schmerz« (2016). 
Neben dem Redaktionsteam sind viele verschiedene Jugendliche Autor"innen im »Milchbüechli«. Manche sind regelmäßig dabei, andere schreiben einmalig fürs »Milchbüechli«. So kommt es auch vor, dass bestimmte Themen öfters behandelt werden und die Meinungen oder Akzentuierungen auseinandergehen. Die Autor*innen sind zumeist mit einem Foto, Alter, Beruf und E-Mail-Adresse und manchmal auch dem Nickname von diversen Dating-Plattformen unter dem jeweiligen Artikel abgebildet. Die Textformen reichen von Berichten, Interviews, Kommentaren, Erfahrungsberichten über Anleitungen, Gedichte, Sprüche bis hin zum »Bullshitbingo«. Viele der Artikel sind mit bunten selbstgezeichneten Bildern und Illustrationen untermalt. Das »Milchbüechli« selbst versteht sich als mehr als eine Zeitschrift: Es ist, eigenen Beschreibungen zufolge, eine Bewegung. ${ }^{111}$ Selbsternanntes Ziel der Milchjugend ist es, "Druck zu machen [...], weil wir nicht einfach sein werden, wie man sein muss «. ${ }^{112}$ Fünf Jahre nach dem Erscheinen der ersten Ausgabe resümiert ein Mitbegründer und ehemaliger Chefredakteur, dass die Zeitschrift der Ort sei, an dem die Jugendlichen sich ein eigenes selbstbewusstes und stolzes Selbstverständnis erarbeitet haben: »Die Zeitschrift wurde zum Ausgangspunkt eines neuen Selbstverständnisses von jungen Menschen, die ihre Sexualität und ihr Geschlecht nicht mehr abhängig von Normen und Vorgaben machen wollen. ${ }^{113}$

Thematisch stehen der Aktivismus, Vielfalt und Sexualität im Zentrum der Milchjugend. Der größte Themenschwerpunkt des »Milchbüechli« in den Jahren 2012 bis 2016 ist der queere Aktivismus. Dieser hat auf verschiedenen Ebenen seinen Raum im »Milchbüechli«. In fast jeder Ausgabe werden queere oder LGBTIQ-Organisationen aus der Schweiz vorgestellt. Dabei handelt es sich zum Teil um Berichte von MilchjugendMitgliedern, die mitunter selbst in anderen Gruppen aktiv sind, oder Interviews mit Aktivist*innen aus anderen Gruppen. Die Milchjugend berichtet und informiert aber auch über die eigenen Projekte, Aktionen und Veranstaltungen. So wird beispielsweise nach jeder »Milchreise« ausführlich über das dort Erlebte berichtet oder es werden dort gestaltete Manifeste veröffentlicht. Die Jugendlichen schreiben von gemeinsamen Aktionen wie einer »Kiss-in«-Demo, einem »falschsexuellen Stadtspaziergang« oder einer »ich oute mich als«-Aktion am Internationalen Coming-out-Tag. Auch von den Erfahrungen und Erlebnissen auf der Pride wird berichtet und die Forderungen der Milchjugend für die Pride werden veröffentlicht. Nicht zuletzt auch, weil die Milchjugend selbst auf jeder Pride in der Schweiz vertreten ist, werden die Pride und aktuelle Pride-Mottos - zum Beispiel die Ehe für alle - besonders häufig diskutiert. Neben dem Aktivismus ist im »Milchbüechli« auch die Darstellung von vergeschlechtlichter und sexueller Vielfalt zentral. Es wird meistens in Form von Erfahrungsberichten und Interviews von Polyamorie, Trans*, romantischen Männer- und Frauenfreundschaften, alternativen Beziehungsmodellen, LGBTIQA und Behinderungen, Asexualität, Bi- und Pansexualität, unterschiedlichen Familienmodellen, Sexarbeiter*innen, Androgynität berichtet. Ein dritter zentraler Aspekt der selbstbewussten Selbstdarstellung der »falschsexuellen« Jugend im »Milchbüechli« ist der offene Umgang mit Sexualität. So

111 Milchjugend: Hauptgang.

112 Milchjugend: Wir machen Druck.

113 Milchjugend: Ausgabe 20. 
berichtet die Milchjugend über Besuche im Sexshop, spricht über Hierarchien beim Analsex, Strap-ons, lesbischen Sex, feministische Pornos, Grenzen, Konsens und Gesundheit oder ruft die »Dildokratie« aus. Auch die Milchstatistik der Jubiläumsausgabe 2017 bestätigt die zentrale Rolle eines selbstbewussten Umgangs mit Sexualität im »Milchbüechli«: »Genitalien dürfen in unserer Welt beim Namen genannt werden. $36 \mathrm{x}$ Penis und 53 x Vagina ergeben 4,7 erwähnte Genitalien pro Ausgabe. «114 Neben diesen drei zentralen Themenschwerpunkten schreiben die "falschsexuellen« Jugendlichen über Themen, die sie in ihrem Alltag beschäftigen: Liebeskummer, Trennung, Comingout-Erfahrungen, den Freund nach Hause bringen, Dating-Plattformen, Liebesgedichte, ihre liebsten »queeren« Musiker*innen, LGBTIQ-Charaktere in Filmen, Serien, Tattoos und vieles mehr.

Das »Milchbüechli« ist mit dem starken Fokus auf den Aktivismus und aktivistische Debatten eine Bewegungszeitschrift, in der eine gemeinsame Haltung entwickelt wird. Im »Milchbüechli« werden aber auch die eigenen Aktionen medial verlängert, resümiert und diskutiert sowie für die eigenen Projekte und Aktionen mobilisiert. Gleichzeitig ist das »Milchbüechli« eine Zeitschrift, in der Jugendliche ihre Erfahrungen austauschen können und alternative, vielfältige Möglichkeiten des Seins eröffnet werden. Die »falschsexuellen« Jugendlichen präsentieren sich gleichzeitig als offene, selbstbewusste, stolze, aber auch widerständige Gemeinschaft. In diesem Sinne ist das »Milchbüechli« in doppelter Hinsicht zentral um die Pride angeordnet: Zum einen ist die Pride das am meisten diskutierte Thema, zum anderen ist die im Kontext der Pride entstandene selbstbewusste Darstellung der eigenen Sexualitäten und Identität das, was im »Milchbüechli« sowohl auf Text- als auch auf Bild-Ebene hergestellt wird. 
